\title{
ELECTROMAGNETIC INTERACTIONS WITH NUCLEONS AND NUCLEI
}

\author{
Progress Report \\ for \\ DOE Grant No. DE-FG05-89ER40501 \\ University of Virginia \\ Principal Investigators: $\quad$ Stephen T. Thornton \\ Richard M. Sealock
}

November, 1990 - October, 1991

Charlottesville, Virginia

October 30.1991 
Table of Contents

Page number

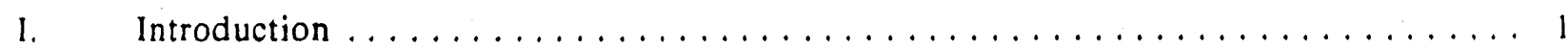

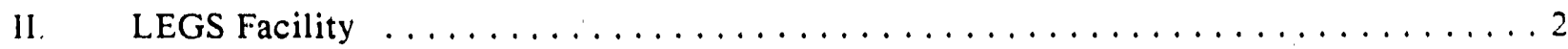

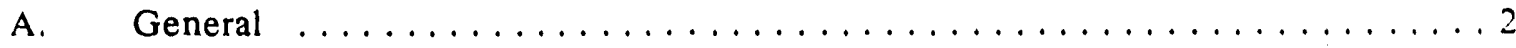

B. $\quad \mathrm{NaI}(\mathrm{Tl})$ Gamma-ray Spectrometer $\ldots \ldots \ldots \ldots \ldots \ldots$

C. Nucleon Detector System $\ldots \ldots \ldots \ldots \ldots \ldots \ldots \ldots \ldots \ldots \ldots$

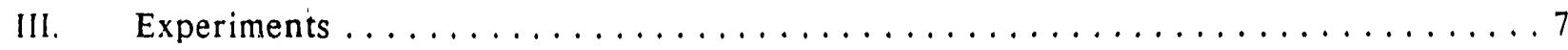

A. Photodisintegration of the Deuteron $\ldots \ldots \ldots \ldots \ldots \ldots \ldots \ldots$

B. $\quad p(\gamma, p) \pi^{\circ}$ and the E2/M1 Ratio in the Excitation of the $\Delta^{+} \ldots \ldots \ldots$

C. $\quad{ }^{3} \mathrm{He}(\gamma, \mathrm{NN}) \ldots \ldots \ldots \ldots \ldots \ldots \ldots \ldots \ldots \ldots \ldots$

D. ${ }^{4} \mathrm{He}\left(\pi^{+}, \mathrm{piN}\right) \ldots \ldots \ldots \ldots \ldots \ldots \ldots \ldots \ldots \ldots \ldots \ldots$

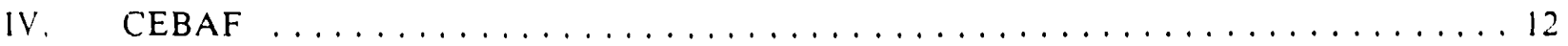

V. Waveshifting Optical Fiber Applications $\ldots \ldots \ldots \ldots \ldots \ldots \ldots$

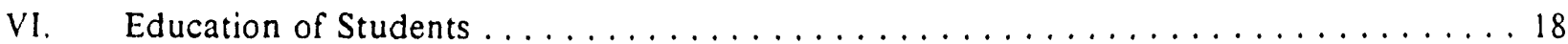

VII. $\quad$ References ................................19

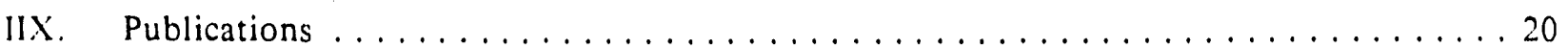

\section{DISCLAIMER}

This report was prepared as an account of work sponsored by an agency of the United States Government. Neither the United States Government nor any agency thereof, nor any of their employees, makes any warranty, express or implied, or assumes any legal liability or responsibility for the accuracy, completeness, or usefulness of any information, apparatus, product, or process disclosed, or represents that its use would not infringe privately owned rights. Reference herein to any specific commercial product, process, or service by trade name, trademark, manufacturer, or otherwise does not necessarily constitute or imply its endorsement, recommendation, or favoring by the United States Government or any agency thereof. The views and opinions of authors expressed herein do not necessarily state or reflect those of the United States Government or any agency thereof. 


\section{INTRODUCTION}

In this document we report the progress for DOE Grant No. DE-FG05-89ER40501. The grant began in March 1991 but we are reporting progress for the period from November 1990 to November 1991.

Our primary research effort continues to be at the LEGS project at Brookhaven National Laboratory. The LEGS facility has made a transition in the last year from a combination of experiment and facility construction to full time experimental work. The first experiment, pinotodisintegration of the deuteron with polarized $\gamma$ rays, is completed and partially published. A second experiment, ${ }^{3} \mathrm{He}(\gamma, \mathrm{NN})$, has also been completed and analysis has begun. We expect to begin two more experiments in the next year.

Other research efforts are primarily related to CEBAF and this work is growing, since major CEBAF experimental equipment is now being constructed. We are heavily involved in the CLAS collaboration in Hall $B$ which will build the CEBAF Large Acceptance Spectrometer. Our contributions to the CEBAF experimental program and equipment construction are described.

The ${ }^{3} \mathrm{He}(\gamma, \mathrm{NN})$ experiment at LEGS was the second use, in a new format, of the University of Virginia Nucleon Detector System. It is now a fully developed system. We also describe a new area of instrument development, applications of waveshifting optical fibers to novel position sensitive detectors for neutral particles.

We will also describe our efforts in the education and training of undergraduate and graduate students. Finally the publications and abstracts are appended. 


\section{LEGS FACILITY'}

\section{A.' General}

Even though LEGS was operational for data taking during the past year, improvements continue to be made to the facility to enhance its performance. The National Synchrotron Light Source had good operational performance during the past year and was only down for brief periods that were planned in advance. We have been pleased with the operation of LEGS, especially since it is such a new facility. We discuss some of the improvements:

\section{Laser - optical system.}

In the past year the method of determining the laser polarization was changed. In the past we had used a simple quartz plate at the $180^{\circ}$ port to reflect laser light both parallel and perpendicular to the experimental reaction plane. The problem was that there were reflections from both the entrance and exit planes of the quartz plate, which could interfere and/or cause alignment problems at the power meter. It was also hard to align the plate to the Brewster angle. The new method replaces the quartz plate with a quartz prism where the entrance and exit angles are not parallel. This serves two purposes. First, only reflections from the entrance plane enter the power meter. Second, the exit angle was chosen to be perpendicular to the refracted laser light and is reflected back through the entrance angle and along the axis of the incoming laser light. This occurs only when the incoming light enters at the Brewster angle, and therefore is very convenient for alignment purposes.

New mirrors were also purchased which will allow the laser to be tuned to the deep-uv region at $300 \mathrm{~nm}$. These mirrors are currently on hand, and we are waiting for the present tube to fail, when both the new mirrors and tube will be installed.

\section{Repeaters}

An additional set of tagger discriminator repeaters wa: installed which carry signals to the second LEGS electronics and data acquisition aren. This suppiies the full set of tagging symbols to the second statio thereby allowing one area to setup and debug electronics parasitically whiie another

area is taking data. The new station was first used during the ${ }^{3} \mathrm{He}(\gamma, \mathrm{NN})$ experiment and performed well.

3. The Laser Gain Stabilization System

During the past few months the laser stabilization and calibration system for use with the large $\mathrm{NaI}(\mathrm{TI}) \gamma$-ray detector at LEGS has been assembled and tested, and is ready to be moved to the LEGS 
experimental area for use. It consists of an Omnichrome He-Cd laser that operates at $54 \mathrm{~mW}$ at 442 $\mathrm{nm}$. The laser light is passed though an opto-acoustical coupler which modulates the light into a pulse with a shape similar to that of the output of the system being stabilized, in the first case a Nal crystal. The pulsed light is then split into 32,64 , or 96 separate lines, which transmit the pulse to the detectors by 100 micron diameter quartz optical fibers. One line is sent into a small 'control' NaI which is near a source and is used to monitor the light output of the laser. The gain of the small Nal is stabilized relative to the source, and the peak due to the laser is then monitored and used as input to a feedback circuit which adjusts the shaper output accordingly. This system has been shown to limit laser output fluctuations to a few percent. Since the centroid of the laser peak will usually be determined to within at least a tenth of the width of the peak, the design goal of $0.5 \% \%$ gain stabilization is easily satisfied. Currently, the sensitivity of the feedback circuit is being optimized to avoid oscillations, and then the entire assembly will be moved to the LEGS experimental area.

\section{B. Nal(Tl) Gamma-ray Spectrometer}

A very large, high resolution NaI (Tl) detector has been constructed as the primary LEGS $\gamma$ ray detector. The large volume of the single NaI ingot, 19" diameter by 19" long, is required to contain the electromagnetic showers of the high energy $\gamma$ rays produced at the LEGS facility, which will ultimately reach $400 \mathrm{MeV}$. A single ingot is desirable because nonuniformities or discontinuities will degrade the resolution. In collaboration with Bicron Corporation, techniques utilizing cosmic-ray muons and computed tomography [1] were developed to evaluate candidate ingots. The detector was delivered to LEGS in 1990. During the past year, work has concentrated on constructing parts for mounting the crystal and its plastic scintillator veto counters and lead shielding.

The detector is now mounted on a temporary goniometer while parts for the final goniometer are being constructed. The crystal can be rotated into the primary photon beam for resolution tests. It will be surrounded by 2 to 4 inches of plastic scintillator, which will be used to identify photons whose showers are not contained in the crystal or events resulting from cosmic ray background. The 2 inch thick front plastic scintillator veto paddle that will be used in the final detector housing is also temporarily installed. Light from the $\mathrm{NaI}$ is collected in 19 Hamamatsu 1911 phototubes powered by high performance bases constructed to minimize any photon flux-rate dependencies. An initial spectrum from the detector system is shown in Fig. 1. The data were taken on Compton backscattered photons produced at LEGS in coincidence with a tagging spectrometer pulse corresponding to a photon energy of $304 \mathrm{MeV}$ and in anticoincidence with the front plastic detector. This yields an energy resolution of $1.7 \%$ FWHM after deconvoluting the energy spread of the stored electron beam and the tagging spectrometer acceptance. Cosmic-ray tomography images combined with EGS4 shower calculations gave a resolution prediction of $2.5 \%$ or better. That is, the measured 


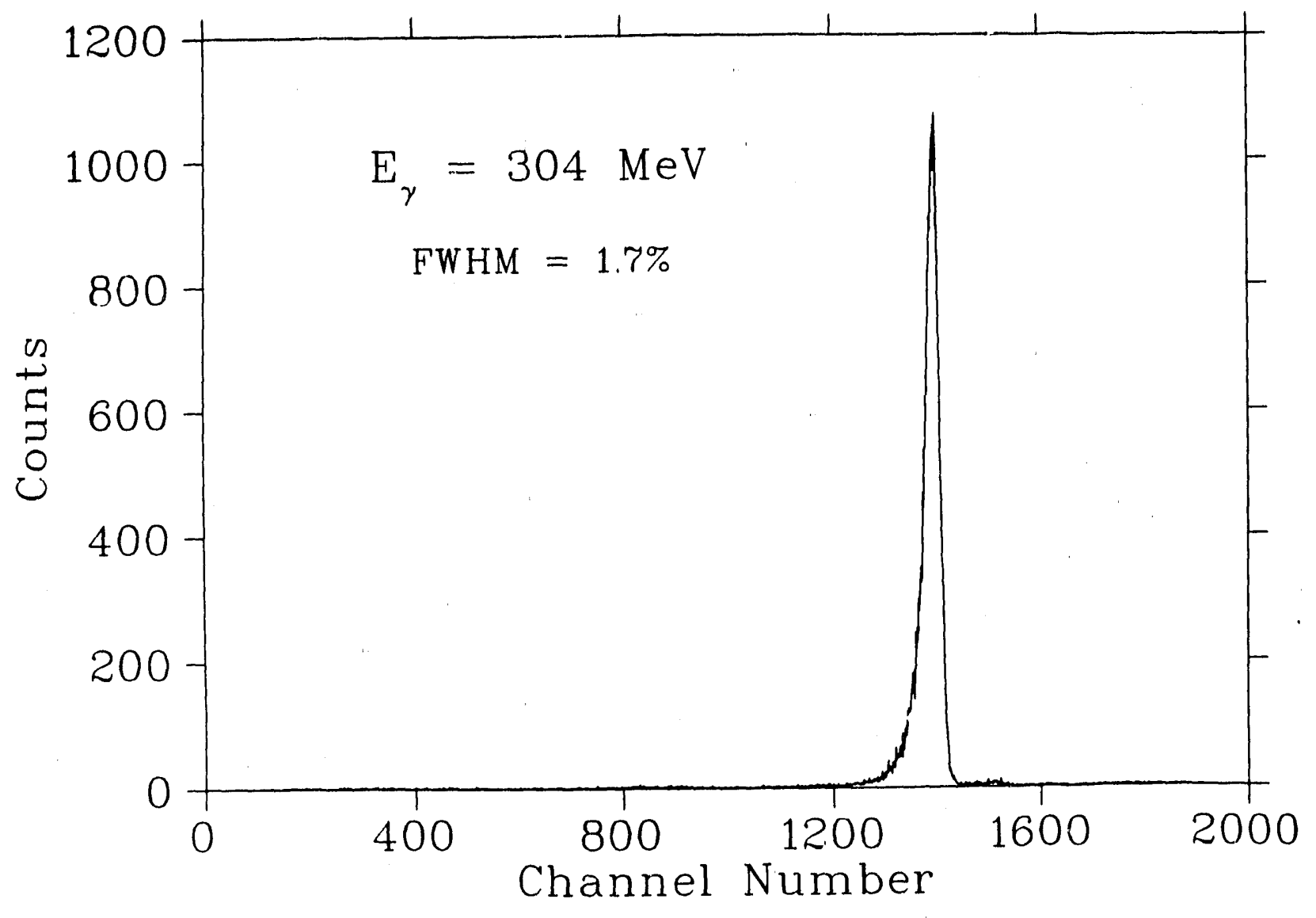

Figure 1. Spectrum obtained with the LEGS 19" $\times 19^{\prime \prime} \mathrm{NaI}(\mathrm{Tl}) \gamma$-ray detector. The incident $\gamma$ rays had an energy of $304 \pm 2.5 \mathrm{MeV}$. A 2" thick plastic scintillator in front of the detector was used to veto events where some of the shower escaped from the front of the detector. The detector resolution is $1.7 \%$ FWHM after deconvoluting the beam energy spread. 
nonuniformities were as large as the uncertainties in the tomography data so that the resolution prediction is an upper limit. The measured energy resolution is expected to improve with further development of phototube gain matching and stabilization and inclusion of the annular plastic scintillator veto detectors.

\section{Nucleon Detector System}

The University of Virginia nucleon detector system has been an important project for the present group during the past few years. The State of Virginia contributed about $\$ 200,000$ towards the purchase of a comprehensive scintillator based, large area, detector system used to detect nucleons, pions, and other light particles. The system was mostly completed a year ago, but improvements to the system have continued. It has now been used in two experiments, a ${ }^{4} \mathrm{He}(\pi, N N)$ measurement at LAMPF in 1990 and a ${ }^{3} \mathrm{He}(\gamma, N N)$ measurement at LEGS in 1991. Additional scintillators, photomultipliers and associated electronics that will double the volume of the array for use at CEBAF are being purchased by the University of Virginia and Basel.

The present system consists of 32 scintillator bars, each measuring $10 \mathrm{~cm} \times 10 \mathrm{~cm} \times 160 \mathrm{~cm}$ and having light pipes and 2 " diameter photomultiplier tubes at each end to collect sufficient light for accurate particle time-of -flight measurements and to enable position determination along the $160 \mathrm{~cm}$ length. Although primarily intended as a neutron detector, the system is also useful for detecting low mass charged particles, such as pions, protons, and deuterons. The large number of bars enables a variety of configurations to be set up with good angular coverage, while still providing goud neutron detection efficiency. The system includes 24 scintillator paddles, each measuring $0.6 \mathrm{~cm} \times 11 \mathrm{~cm} x$ $160 \mathrm{~cm}$, to provide charged particle identification. When the system is used to detect charged particles, the paddles reduce accidental rates and provide $\Delta E-E$ particle identification. For neutron detection the paddles act as charged particle vetoes.

The two primary quantities measured with a TOF detector system are the flight time of the particle and the position location in the detector. The specifications of the system are determined by the ciesired energy and angle resolutions. For a fixed flight path and a given particle energy (i.e. fixed flight time $t$ ) the energy resolution improves with decreasing flight path uncertainty, $\Delta \mathrm{l}$, and decreasing time dispersion, $\Delta t$, of the TOF detector. Thus good energy resolution can only be obtained with small intrinsic time uncertainties in individual bars and for small bar dimensions in the particle direction. The event location along a bar is derived by the time-difference method, while the position in the other dimension, for neutrons, is determined from the bar position in the counter matrix. Large area coverage with good resolution in both dimensions is thus achieved by using a large number of long, narrow bars, together with good timing precisinn at both ends of each bar. 
Neutron efficiency increases by about $10 \%$ for each additional $10 \mathrm{~cm}$ of scintillator. Good efficiency $(>10 \%)$, therefore, requires a large amount of material for the neutrons to pass through. Good time resolution, on the other hand, favors thin bars, so that good neutron efficiency and good time resolution would require an expensive array with a large number of PMT's and related electronic components.

The choice of the bar size, $10 \mathrm{~cm} \times 10 \mathrm{~cm} \times 160 \mathrm{~cm}$, is a good compromise for our experiments. The square cross section allows flexibility as well as ease in the mechanical construction of apparatus to hold the detector system in the desired configuration which can be either horizontal or vertical with respect to the beam line. The bars can be used in either of the two configurations for detection of protons and neutrons simultaneously. A layer of scintillator $30 \mathrm{~cm}$ thick (three bars) can stop protons of energies up to $200 \mathrm{MeV}$, while a $10 \mathrm{~cm}$ thick layer (one bar) can stop protons of energies up to $100 \mathrm{MeV}$. Actual proton energy is determined from the TOF measurement from the ta'get to the bar.

We have chosen Bicron $\mathrm{BC} 408$ scintillating material for both the bars and paddles, because of its short rise time $(0.9 \mathrm{~ns})$, short pulse width (2.5 ns FWHM) and long light attenuation length (210 $\mathrm{cm}$ ). The light guides placed at each end of the bars and paddles are made of UVT acrylic lucite, machined and polished in single pieces.

We have chosen the Amperex XP2262 photomultiplier tube for use with our bars and paddles. The entire nucleon detector system requires two tubes for each bar and paddle for a total of 144 tubes. The XP2262 is a 12-stage, $44 \mathrm{~mm}$ useful diameter head-on tube type with a plano-concave window. Its photocathode is semi-transparent type $\mathrm{D}$ with high cathode sensitivity. It has very good linearity and time characteristics. Its anode pulse rise time (at $2200 \mathrm{~V}$ ) is $2.0 \mathrm{~ns}$ with a total time throughput of $30 \mathrm{~ns}$.

We have chosen the CERN photomultiplier tube base, model 4244. Current independent voltages are supplied to the last three dynodes by high voltage zener diodes to stabilize the voltage. The printed circuit, including all components and output connections, is fitted directly on the tube base socket. Because of the large number of photomultiplier tubes used, we are using the LeCroy model 1440 high voltage power supply system.

We have chosen to make maximum use of Emitter Coupled Logic (ECL) electronic modules, such as provided by LeCroy. Considerable economy in space, setup time, and cost is achieved because large numbers of circuits are combined into a small space. The circuits are easily connected in units of 16 with flat ribbon cable. Rather than coaxial cable, ECLine uses twisted pairs, and complementary logic is easily obtained by reversing the two leads in a pair. The price per channel is substantially less than with standard NIM electronic modules. We make use of the following electronic units: 
- The pulse splitters are simple resistive networks.

- The discriminators are 16 channel devices (LeCroy 4413) that produce an output when the input pulse height reaches a preset level. The threshold settings are programmable via CAMAC. The discriminators use 50 coaxial connectors at the inputs, and ECLine ribbon connectors at the outputs.

- Five ECLine Logic Units (LeCroy 4516) are used to form a coincidence between the two ends of each bar and paddle. Each has 16 channels with each channel capable of performing any combination of AND/OR (and complements thereof) logic on three input pulses. These are CAMAC units which can be controlled and interrogated by the control computer.

- The time of each signal at the output of the discriminators is measured using a CAMAC Time-Digital-Converter (TDC).

- The height of the pulse from each photomultiplier tube is measured in a CAMAC Analog-Digital-Converter (ADC) (LeCroy model 4300B/610).

The signal from each photomultiplier tube anode is split: one signal is sent to the ADC to digitize its amplitude while another is sent to a discriminator. One discriminator output goes to a TDC, while another goes to an ECLine Logic Unit with 16 channels of three-fold AND/OR logic. Each channel in this unit can be used to form either an AND or an OR of the signals from the two ends of a bar or paddle. The ECL Logic Unit includes a NIM standard output which is the logical OR for all sixteen logic circuits. This 16 -fold OR is combined for all three Logic Units serving the 48 bars in a NIM standard OR circuit. Similarly the OR signals from the paddles can be combined into a NIM standard OR circuit if desired. The OR of all 48 bars is put into coincidence with signals from other parts of the experiment to form an event trigger. The paddle OR can play a part in the event trigger if desired. The event trigger signal provides the START signal for the TDC's used to measure the time of a pulse in any bar or paddle. The width of the event trigger can be adjusted to make it usable as a GATE signal for the ADC's.

So far two separate detector holders have been constructed. The first one supports the paddles and bars in a vertical mode that can be arranged in any desired angle pointing towards a common target. We have used this system for an experiment at LAMPF in 1990. The other configuration has the paddles and bars in a horizontal direction placed above the target in a semicircle. 1 his configuration was used during 1991 in the ${ }^{3} \mathrm{He}$ experiment at LEGS. 


\section{EXPERINENTS}

\section{A. Photodisintegration of the Deuteron}

The first experiment to be performed at LEGS was photodisintegration of the deuteron, $D(\gamma, p) n$, with polarized gamma rays. This experiment began in December of 1989 and was continued, with many interruptions for facility development, until March 1991. This experiment has been a success and has demonstrated that LTGS is now a working facility. Some of the data have been analyzed while the remaining analysis is ongoing. Initial results from a subset of the data have already been published in Physical Review Letters [2]. A copy of this letter is included in the section on publications. Additional preliminary results are presented here and will be published soon.

The experiment used a liquid deuterium target and two independent proton detectors. An array of $21 \mathrm{CaF}_{2}$-plastic-scintillator phoswich detectors at seven angles were used for tagged photons with energies above $190 \mathrm{MeV}$. Protons were clearly identified in a plot of energy loss in the $\mathrm{CaF}_{2}$ versus total energy deposited in the $\mathrm{CaF}_{2}$ and plastic. The second set of proton detectors consisted of four planes of silicon microstrips followed by a thin plastic scintillator to measure energy loss and a $25 \mathrm{~cm}$ deep, $\mathrm{NaI}(\mathrm{Tl})$ detector for total energy measurement. The silicon microstrips provided tracking resolution of $0.3^{\circ}$ which allowed identification of background events from target windows. This detector system was used at three angles and for untagged photons in the energy interval from 105 to $304 \mathrm{MeV}$.

Data were collected in several periods using different configurations of the tagging focal plane detector array and two different wavelengths of the initial laser photons. The main data taking periods that produced high quality data were May to June 1990 and January to March 1991 for the low and high energy data sets, respectively. The two laser wavelengths were $488 \mathrm{~nm}$ (visible) and 351 $\mathrm{nm}$ (UV), giving maximum $\gamma$-ray energies of 227 and $304 \mathrm{MeV}$, respectively. Polarization of the $\gamma$ ray beam was flipped randomly between being parallel and being perpendicular to the reaction plane. The enersy resolution of the $\gamma$-ray beam was typically $5 \mathrm{MeV}$. Background yields from bremsstrahlung $\gamma$ rays were measured regularly.

Data from the two detector systems were analyzed independently which resulted in a good check of systematic errors. Analysis of the lower energy data set is complete. Analysis of the UV data is underway, and there ara no preliminary results available yet.

The published data were a selection of the data obtained with $488 \mathrm{~nm}$ laser light. Further data from this run are shown in Fig. 2 - 5 for both the microstrip and phoswich detector systoms. Figure 2 shows differential cross sections, $d \sigma / d \Omega$, at three center of momentum angles. Filled circles indicate data from phoswich detectors and the stars indicate data from the microstrips. Figure 3 shows the 


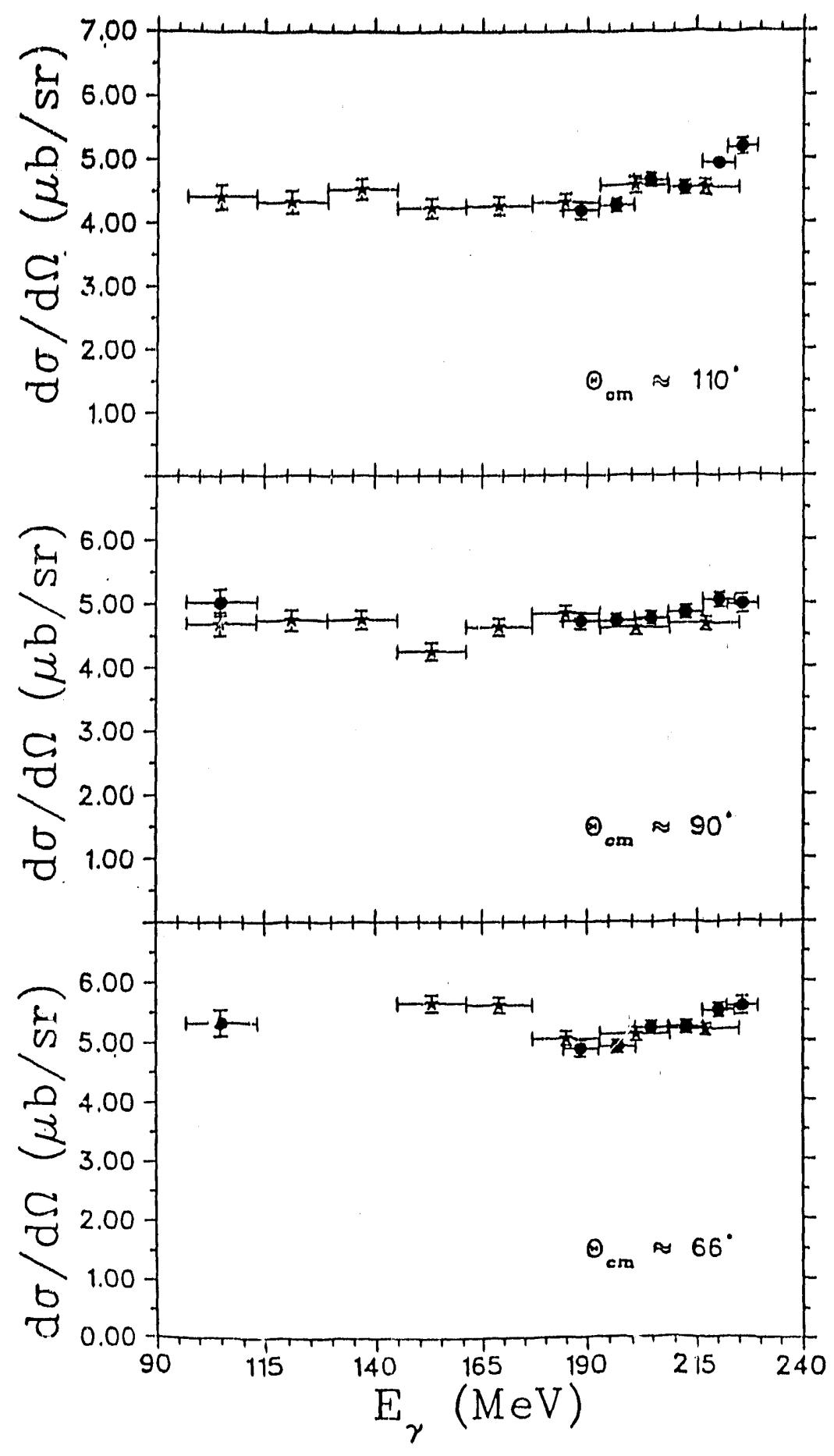

Figure 2. Differential cross sections versus $E_{\gamma}$ for $D(\gamma, p)$ n measured at $\theta_{c m}=66^{\circ}, 90^{\circ}$ and $110^{\circ}$ with a phoswich array (filled circles) and silicon r.icrostrips (stars). 


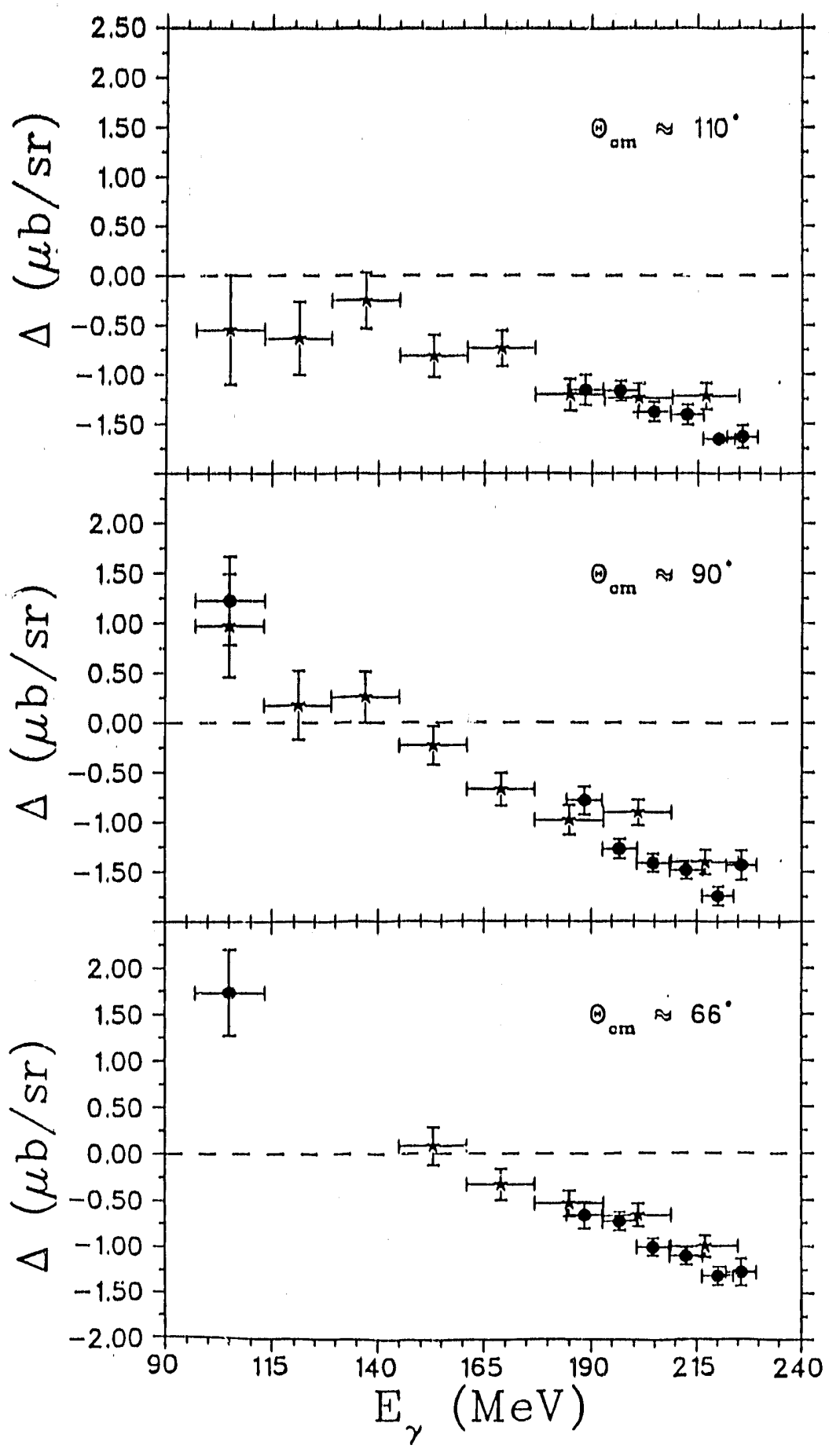

Figure 3. The polarization difference, $\Delta$, defined as $\sigma_{1}-\sigma_{\perp}$. Filled circles indicate data from phoswich detectors and the stars indicate data from the silicon microstrips. 


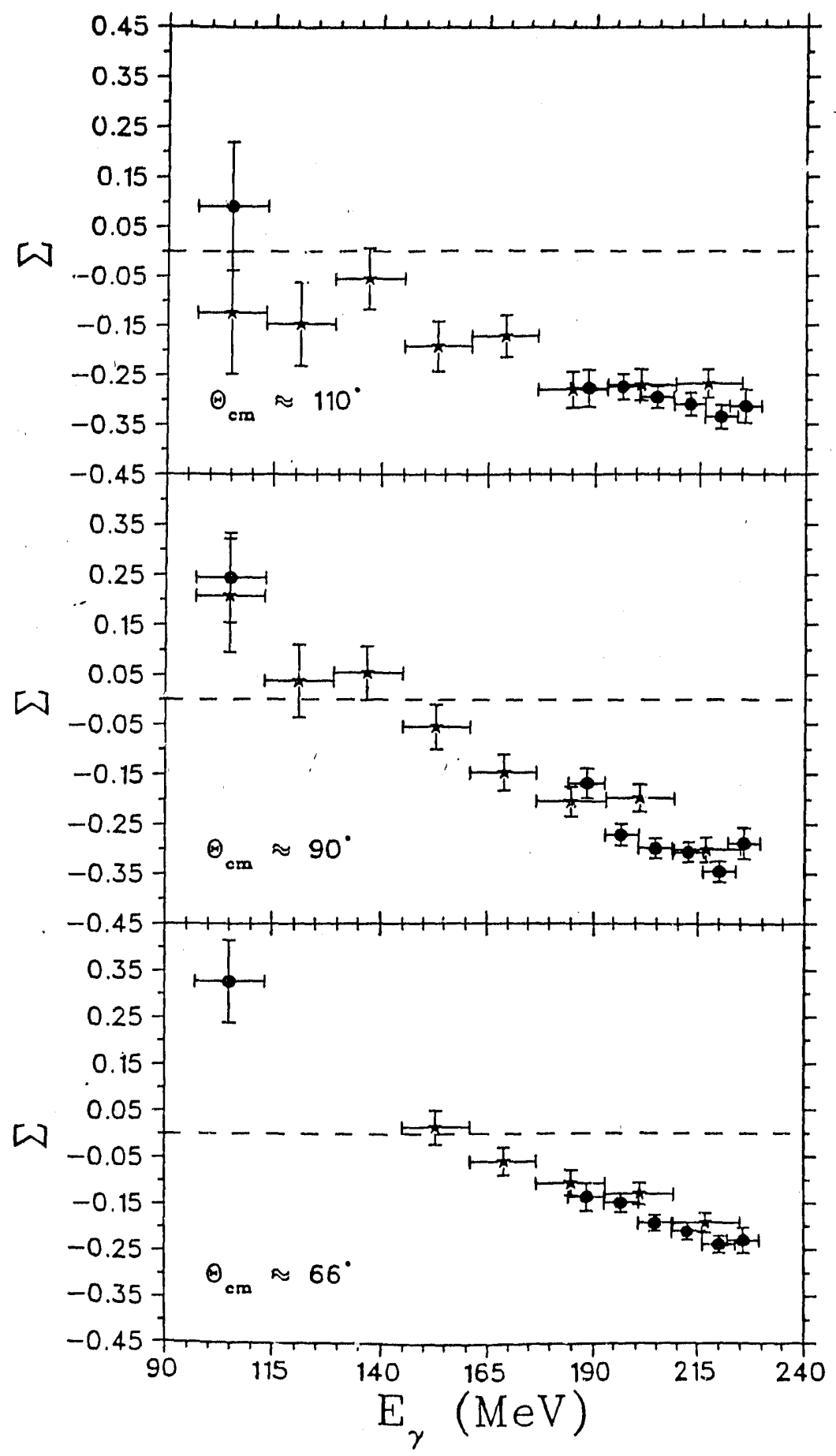

Figure 4. Photon asymmetry, $\Sigma$, which is proportional to $\Delta$ divided by $\mathrm{d} \sigma / \mathrm{d} \Omega$. Filled circles indicate data from phoswich detectors and the stars indicate data from the silicon microstrips. 


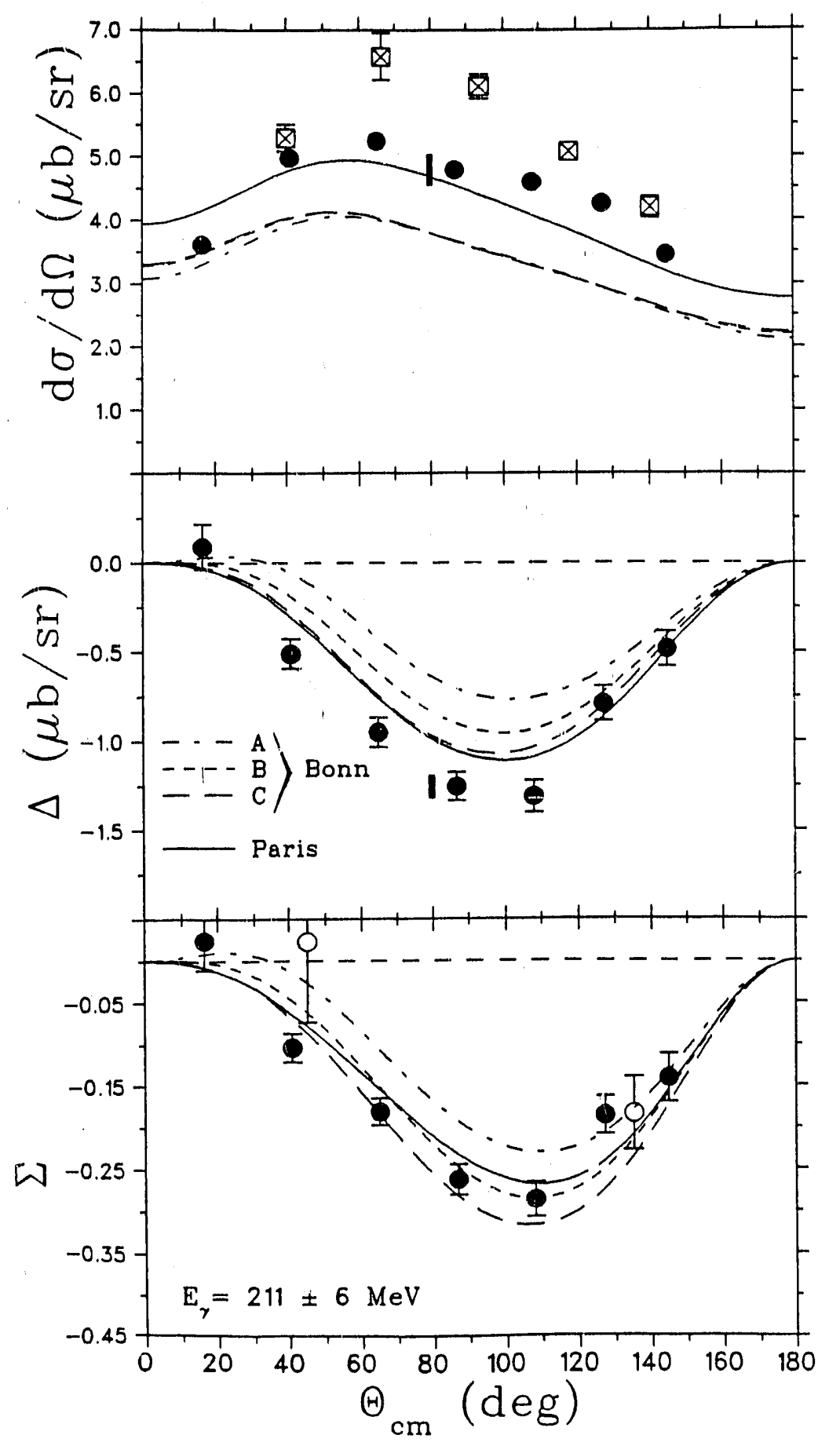

Figure 5. Differential cross sections, $d \sigma / d \Omega$, polarization differences, $\Delta$ and photon asymmetries, $\Sigma$, measured at $E_{y}=211 \pm 6 \mathrm{MeV}$. The solid circles indicate data from LEGS. The crossed squares indicate data from references 4 and 5 , while the circles indicate data from reference 6 . The curves result from calculations by Wilhelm, Leidemann and Arenhoeval [3]. See text for details. 
quantity $\Delta$, defined as the difference between the cross sections with the $\gamma$-ray polarization parallel, $\sigma_{\|}$, and perpendicular, $\sigma_{\perp}$, to the reaction plane. Figure 4 shows the photon asymmetry, $\Sigma$, which is the ratio of $\Delta$ and $d \sigma / d \Omega$. Angular distributions of $d \sigma / d \Omega, \Delta$ and $\Sigma$ at a $\gamma$-ray energy of $211 \mathrm{MeV}$ are shown in Fig. 5 along with calculations of these quantities by Wilhelm, Leidemann and Arenhoevel [3]. The calculations used the Paris potential and three versions of the Bonn potential which differ in their treatment of the $N N \pi$ vertex form factor. Also shown in Fig. 4 are previously published data points from DeSanctis et al. [4], Levi Sandri et al. [5] and Liu [6]. There are no other data for $\Delta$ because other laboratories have not independently measured $\sigma_{1}$ and $\sigma_{\perp}$.

It is clear that $\Delta$ contains the greatest sensitivity to details of the nucleon-nucleon potential. Fig. 5 shows very good agreement between the measured photon asymmetry and the calculations using the Paris potential yet there is a clear discrepancy in the case of $\Delta$. Other LEGS data ${ }^{2}$ show that this discrepancy grows as the photon encrgy increases. Thus we believe that the high quality of the LEGS data will prove very valuable in constraining models of the short range nucleon-nucleon force.

\section{B. $\quad p(\gamma, p) \pi^{\circ}$ and the E2/M1 Ratio in the Excitation of the $\Delta^{+}$}

A long outstanding question in nuclear physics has been whether there is a $\mathrm{D}$ state component of the nucleon. From the initial proposal the LEGS group has intended to make measurements to search for a $\mathrm{D}$ state component. Compton scattering from the nucleon is one reaction that is sensitive to it. This reaction has been a primary motivation for the construction of the high resolution $\gamma$-ray detector described below. Another candidate reaction is $p(\gamma, p) \pi^{\circ}$, and it was chosen as the first experiment at LEGS to investigate the quadrupole component of the nucleon wave function. This reaction has smaller nonresonant contributions than the charged pion channel, as is shown in the comparison of the total photoabsorption cross sections in Fig. 6. The large nonresonant terms in the charged pion channel are indicated by the dashed curve.

Nonresonant contributions must be determined within the context of a given model. The leading terms contributing to the $\pi^{\circ}$ channel as described in the model by Nozawa, Blankleider and Lee [7] are shown in Fig: 7. In this model the form factor for the resonant $\gamma-p$ vertex is parameterized by an effective cutoff mass, $\Lambda$, and the electric and magnetic coupling constants. The existing pion scattering phase shift data along with the measured unpolarized pion photoproduction data are then used to determine the $\mathrm{El}^{+}(3 / 2)$ nonresonant terms and the above parameters are then adjusted to fit the measured multipole data. The results of these fits are shown in Fig. 8 for the real and imaginary $\mathrm{El}^{+}(3 / 2)$ amplitudes. The data are the result of an nergy-dependent multipole decomposition of the available pion photoproduction data [8]. The contributions of noniesonant terms, labeled "Born", which are determined by the above model are shown by the dashed curve. The resonant terms, shown by the dot-dash curve, are then adjusted such that the sum of the resonant and 

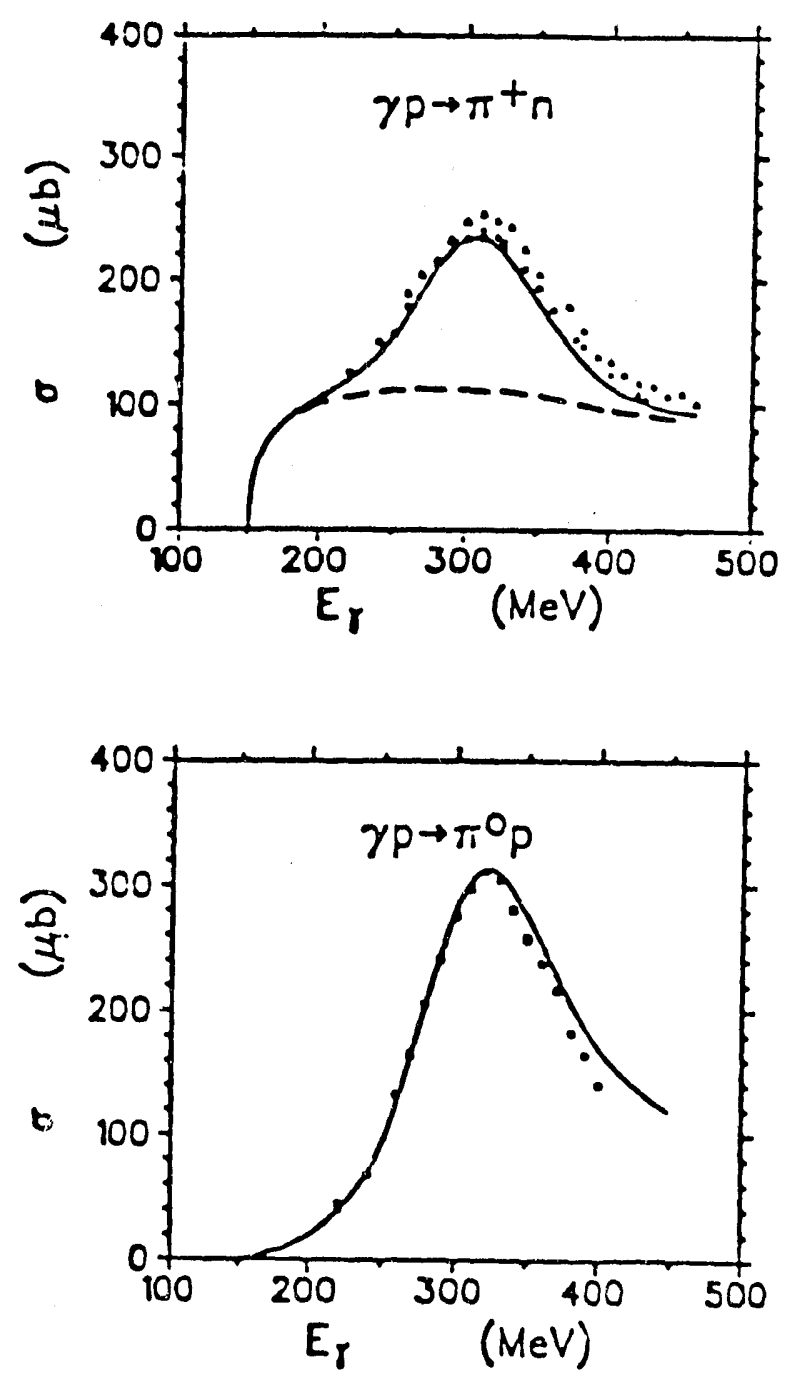

Figure 6. Total photon absorption cross section versus $\gamma$-ray energy for $\pi^{+}$and $\pi^{\circ}$ production. The nonresonant part is indicated by the dashed line. 


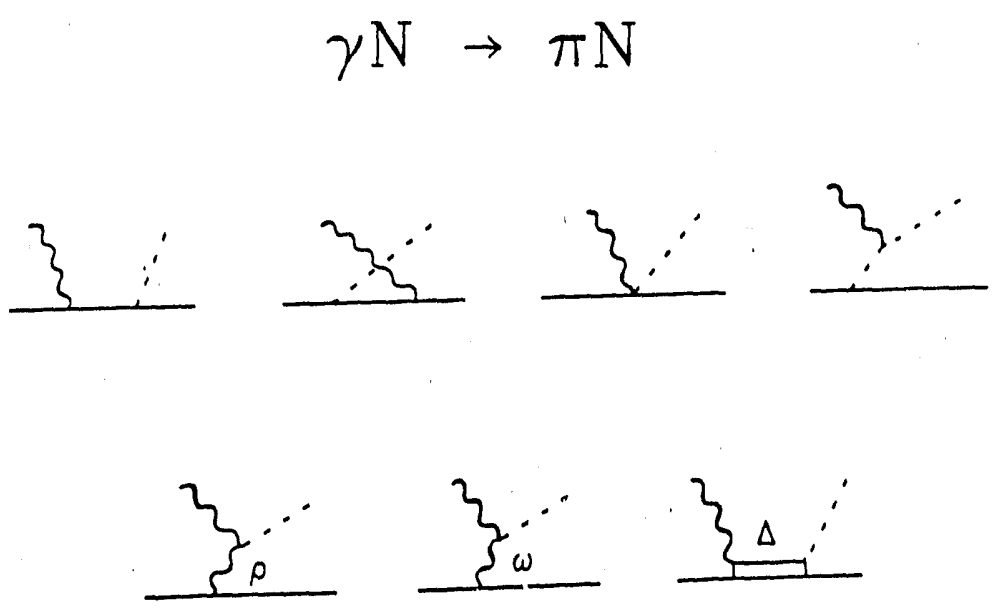

Figure 7. Diagrams contributing to the $\pi^{\circ}$ channel as described in the model of Nozawa [7]. 

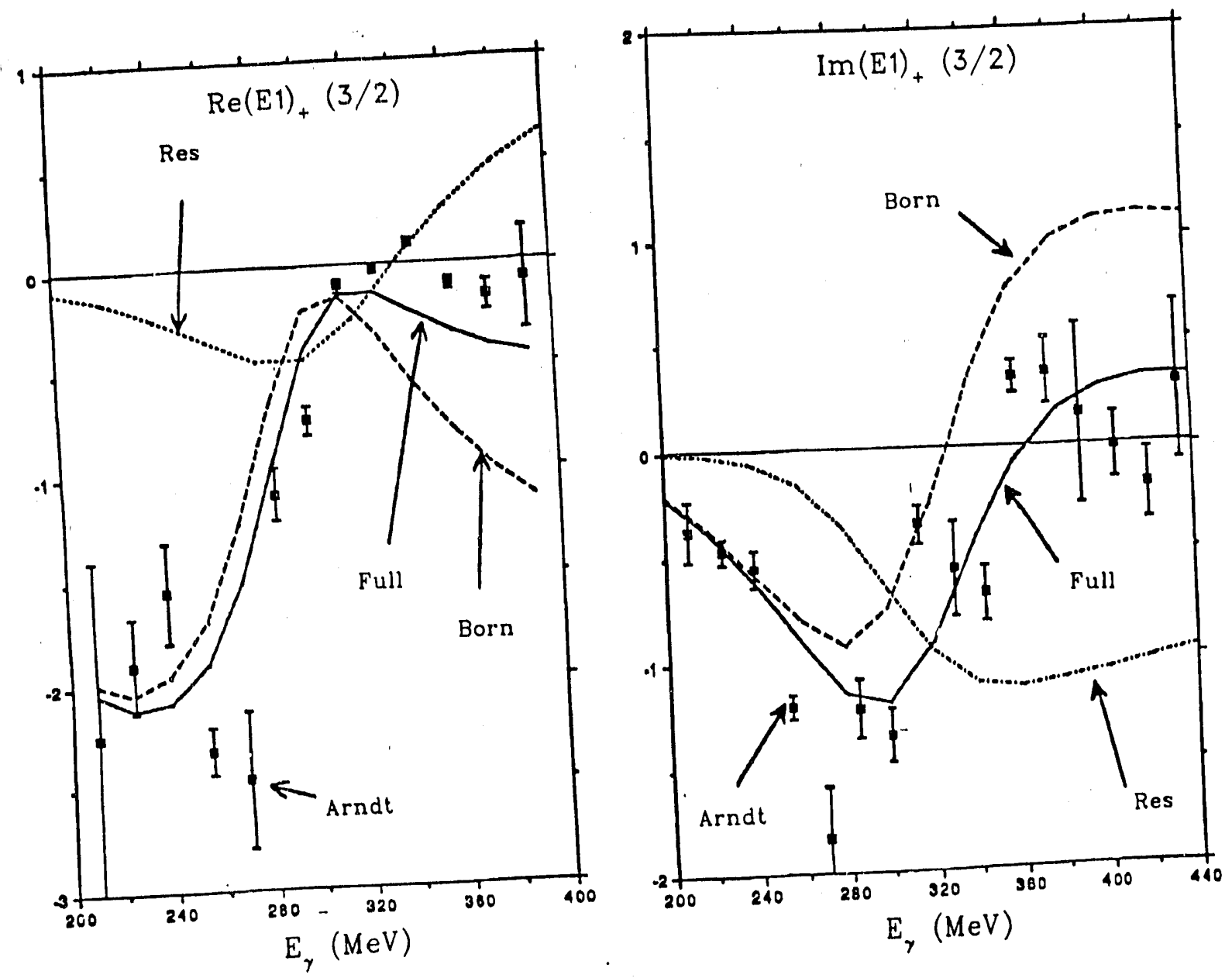

Figure 8. Fits to the pion scattering phase shifts for the real and imaginary parts of the $\mathrm{E}^{+}(3 / 2)$ amplitudes. 
nonresonant terms fit the experimental data. Clearly, the quality of the multipole data is poor and more experimental data are needed to better constrain these models. Also, since the nonresonant terms are large, good measurements of a number of different reaction channels are needed to determine the resonant piece of the delta excitation in a model-independent fashion.

The first data on $\pi^{\circ}$ photoproduction on the proton at LEGS were taken in late 1990 between measurements of the photodisintegration of the deuteron. The same experimental setup as for the deuterium experiment was used, and the target was simply filled with liquid hydrogen. The incident photon energies were tagged from 210 to $315 \mathrm{MeV}$. It is the goal of an experiment planned for autumn 1991 to extend this energy range over the peak of the delta. The cross section is very forward peaked in the lab system, so only the phoswich detectors at angles of $7^{\circ}, 15^{\circ}$ and $35^{\circ}$ were used, which correspond to $105^{\circ}, 122^{\circ}$, and $150^{\circ}$ in the center of momentum system. The silicon microstrip detectors were positioned at $35^{\circ}$.

The ratio of pion photoproduction cross sections for incoming photon polarization parallel and perpendicular to the reaction plane are shown in Fig. 9. These data are preliminary and not for quotation. The ratios at 150 degrees contain data from both the phoswich and microstrip detectors. The curves are from the calculations by Nozawa et al. as described above. The solid curve includes a $3 \%$ E2 contribution to the delta excitation and the dashed curve has no E2 contribution, resonant or nonresonant. Clearly, no conclusion can be made about the E2/M1 ratio using this model with our data since the model fails even to account for the general energy dependence of the data. At this point it is perhaps better to compare the predictions for the unpolarized and polarized cross sections. These are shown for $122^{\circ}$ in the center of momentum system in Fig. 10. For the unpolarized cross sections, the open boxes are data from Bonn [9] and the crosses are data from Lund [10]. The LEGS data is shown as a solid dot in all figures. As the Bonn data set is quite large, it has clearly dominated the multipole analyses and heavily constrained the model, which clearly agrees with the Bonn unpolarized data. We, however, agree with the Lund data and not Bonn, although both sets agree above $340 \mathrm{MeV}$. The perpendicular cross section is very insensitive to the $\mathrm{E} 2$ component and almost entirely determined by the M1 strength. The fact that the model misses the data here is very disturbing, and indicates that even the Ml contribution to this resonance, long taken for granted, may be incorrect. The top graph, which shows the parallel polarization cross section has contributions from both the M1 and E2 multipoles, and the fact that it reproduces the data is likely to be fortuitous.

In conclusion, we have measured the polarized photoproduction of neutral pions on the proton at $105^{\circ}, 122^{\circ}$ and $150^{\circ} \mathrm{cm}$ between 250 and $315 \mathrm{MzV}$. This data, originally intended as a feasibility study, is unlikely to determine the E2/MI mixing ratio in the excitation of the delta. However, and perhaps more importantly, the data does bring into question the dominant $\mathrm{Ml}$ contribution to the delta excitation, as inferred from the polarization observables. This demonstrates the value of the 

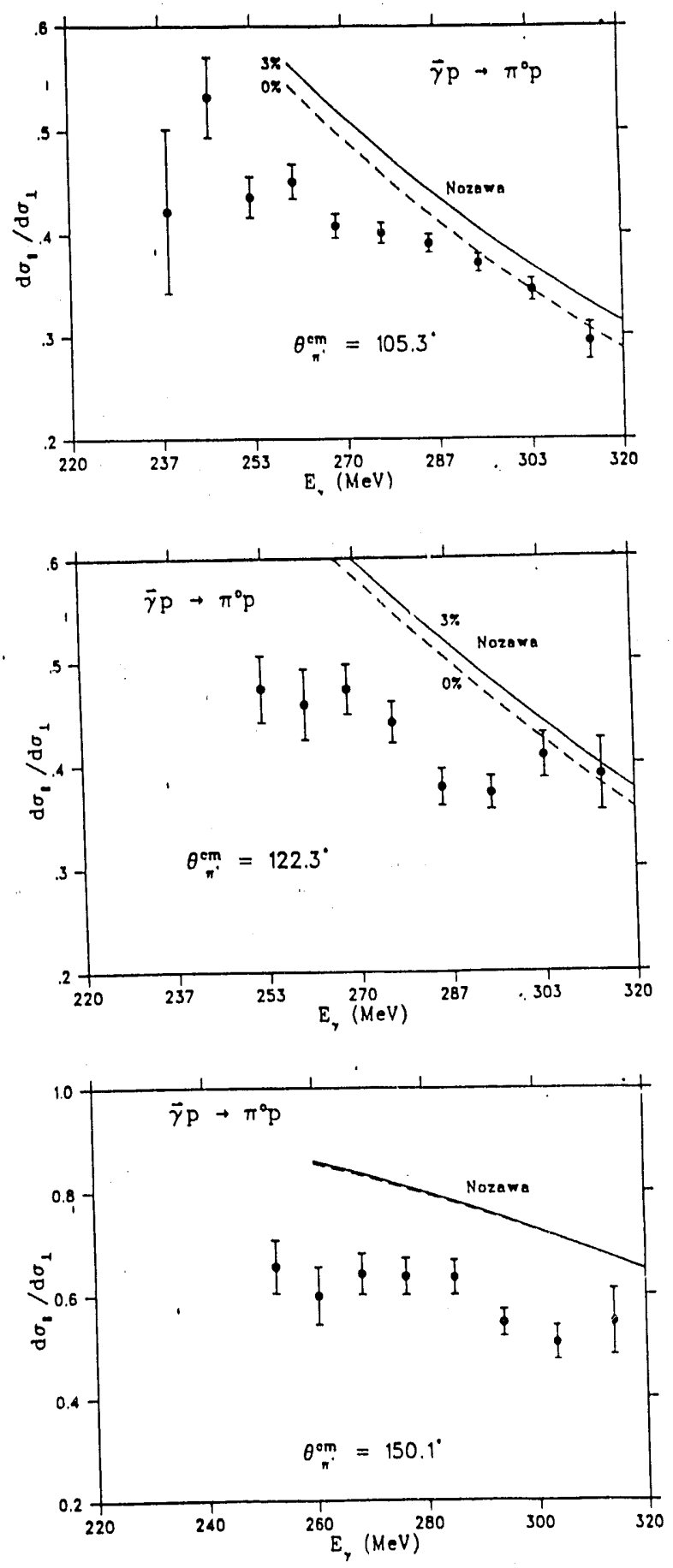

Figure 9. Ratios of pion photoproduction cross sections for incident $\gamma$-ray polarization parallel, $\sigma l$, and perpendicular, $\sigma_{1}$, to the reaction plane. LEGS measurements Caiculations by Nozawa with and filled circles. The data are preliminary and not for quotation. Caiculations by
without a $3 \%$ E2 contribution are shown by the solid and dashed lines, respectively. 

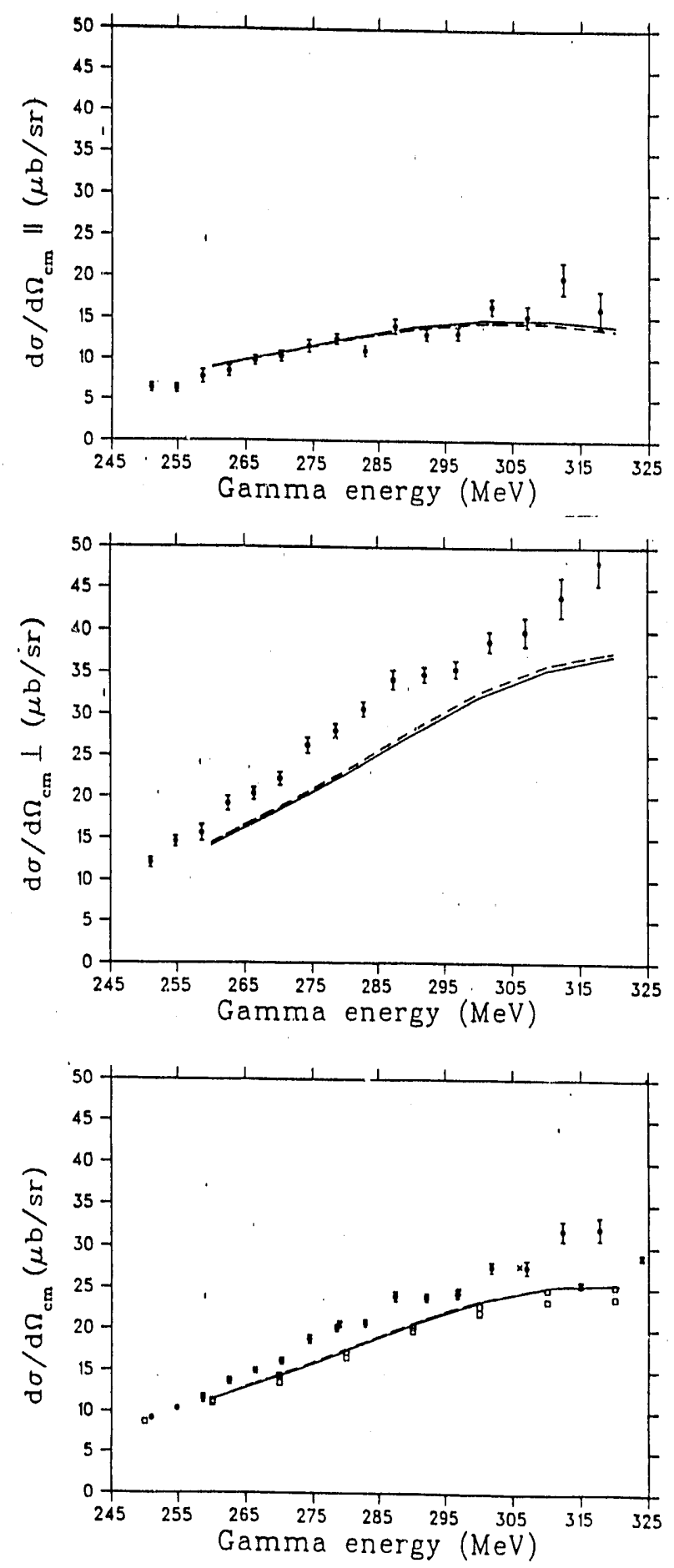

Figure 10. Differential cross sections, perpendicular cross sections and parallel cross sections measured at LEGS, solid circles. Also shown are measurements made at Bonn [9], squares, and Lund [10], x's. Calculations by Nozawa with and without a $3 \%$ E2 contribution are shown by the solid and dashed lines, respectively. 
high quality polarized photon measurements made at LEGS. The current experiment schedule for LEGS calls for this experiment to be carefully repeated in the autumn of 1991 with $\gamma$ rays of energy up to $380 \mathrm{MeV}$.

\section{C. ${ }^{3} \mathrm{He}(\gamma, \mathrm{NN})$}

We recently finished the second complete experiment, ${ }^{3} \mathrm{He}(\gamma, N N)$, at LECS. Set up of the experiment began when the deuteron photodisintegration experiment finished in March 1991. A second data acquisition area was created at LEGS so that this experiment and future experiments would not interfere with the primary electronics setup. Data taking began in August and was finished in September of 1991. To detect coincident outgoing particles two complete detector arays were used - the LEGS phoswich array and the University of Virginia nucleon detector system. An experiment like this uses the full capabilities of the LEGS facilities and requires a lot of manpower.

The motivation for this experiment follows. It is currently believed that two-body correlations, along with meson exchange and delta effects, play an important role in nuclei. ${ }^{3} \mathrm{He}$ (along with ${ }^{3} \mathrm{H}$ ) is the simplest nucleus, other than the loosely bound deuteron, where nuclear effects can be tested. Cross section measurements made with polarized photons will be especially useful in testing theoretical calculations. Recent calculations by J.M. Laget include one-body and two-body currents using the Faddeev wave functions. His calculations have described currently available data fairly well. Our new data will be a more stringent test and allow more discrimination between competing effects. The only known data using polarized photons are some sparse data on ${ }^{3} \mathrm{He}(\vec{\gamma}, \mathrm{p}) \mathrm{d}$; the theoretical calculations are not in good agreement with the data.

Laget's model is not the only theory which can be applied to this reaction, but it emerges as a viable tool for predicting the behavior of the meson exchange and delta currents. For example, Laget predicts a negligible contribution to the $D(\vec{p}, \gamma)^{3} \mathrm{He}$ reaction from one-body currents, even when initial state interactions are included. Although several analyses have been performed for the deuteron photodisintegration, there have been no predictions for ${ }^{3} \mathrm{He}$, which should perhaps be more interesting, because of the two-body currents, meson exchange, and delta effects.

The photodisintegration of the diproton, studied with the ${ }^{3} \mathrm{He}(\gamma, \mathrm{pp})$ reaction where the two protons are detected at the conjugate angles satisfying two-body kinematics, will be very interesting. Since the two protons are identical, the dipole absorption contribution is strongly suppressed, and charged mesons cannut be exchanged between them. Calculations show that cross sections for the ${ }^{3} \mathrm{He}(\gamma, \mathrm{pp})$ reaction should be $5 \times 10^{-3}$ of those expected for ${ }^{3} \mathrm{He}(\gamma, \mathrm{np})$. However, the few existing measurements of the ${ }^{3} \mathrm{He}(\gamma, \mathrm{pp})$ cross sections indicate they are an order of magnitude larger than expected. No data yet exists with polarized photons, and the coincidence experiments should prove very fruitful. 
The coincidence measurements of $p p$ and $p n$ in the outgoing channels represent an experimental challenge. There have been suggestions that the large yields measured in the tails of the quasideuteron absorption peak are evidence of large three-nucleon absorption amplitudes. Since the $\Delta$ amplitudes play an important role in both pion and photon absorption at our energies, we can look for similar effects in the tails of the photon quasideuteron peak. The $(\gamma, p p)$ channel is a good place to look, because the two-body amplitudes are much larger in the $(\gamma, p n)$ channel. The photon asymmetry will be a valuable tool for disentangling the effects of three-body absorption from those of final state interactions and other sequential processes, such as $(\gamma, p n)$ followed by charge exchange on the outgoing neutron.

The experiment utilized the existing Phoswich proton deteciors which consist of $1-2 \mathrm{~mm}$ thick $\mathrm{CaF}_{2}$ followed by thick scintillators. The Phoswich detectors were located below the ${ }^{3} \mathrm{He}$ target, and the UVa nucleon detector system (described clsewhere) were placed in an arc above the target. They consist of a thin scintillator paddle followed by two $10 \mathrm{~cm}$ thick plastic scintillator bars. The paddles and bars are $10 \mathrm{~cm}$ wide and $160 \mathrm{~cm}$ long. The entire system allowed us to take singles and coincidence data simultaneously. Both the Phoswich and nucleon detectors were used to detect charged particles, and for the pn coincidence experiment, the nucleon detectors also detected the neutrons.

The experimental data taking has been completed, and the data are presently being analyzed. In Fig. 11 we show a $\triangle E-E$ spectra from one phoswich detector. The proton band is clearly visible. The proton identification spectrum for the previous figure is shown in Fig. 12. There is no difficulty in identifying the protons. We show in Fig. 13 the corresponding proton peak from the nucleon detector system. The proton peak is isolated very cleanly from background effects. Finally, we show in Fig. 14 the photon tagger spectrum. This spectrum shows the distribution of photon energies over a wide energy range up to $300 \mathrm{MeV}$. Each photon initiating a nuclear reaction is identified by its photon energy.

The preliminary analysis indicates that we obtained sufficient coincidence data to investigate the theoretical questions posed above. Coincidence experiments like this require a large amount of effort to prepare the data into a suitable form for both understanding and presentation.

\section{D. ${ }^{4} \mathrm{He}\left(\pi^{+}, \mathrm{pN}\right)$}

In the summer of 1990 we participated in an experiment at LAMPF to measure pion two-body absorption in ${ }^{4} \mathrm{He}$. Motivations for the experiment were to look for binding energy dependence of the two-nucleon mechanism and to look for $\Delta-\Delta$ induced four-body absorption. Positive pion beams with energies of $500 \mathrm{MeV}$ were used. Outgoing protons were detected in a spectrometir and both 


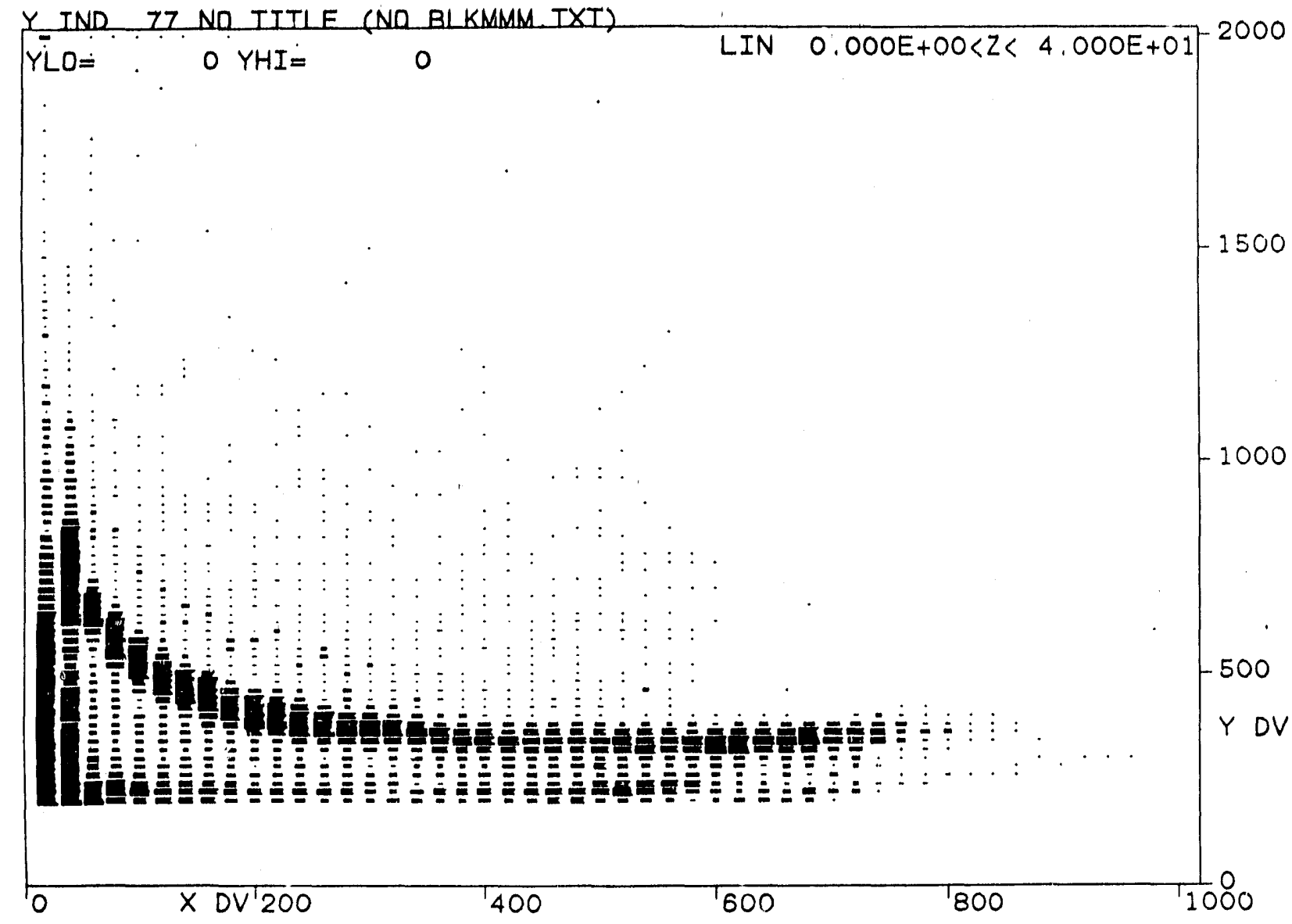

Figure 11. A $\triangle E-E$ plot from one of the phoswich detectors. Protons are clearly concentrated in the dark band. The target was ${ }^{3} \mathrm{He}$. These data were accumulated during one fill of the Light Source storage ring and are only a very small part of the total data set. 


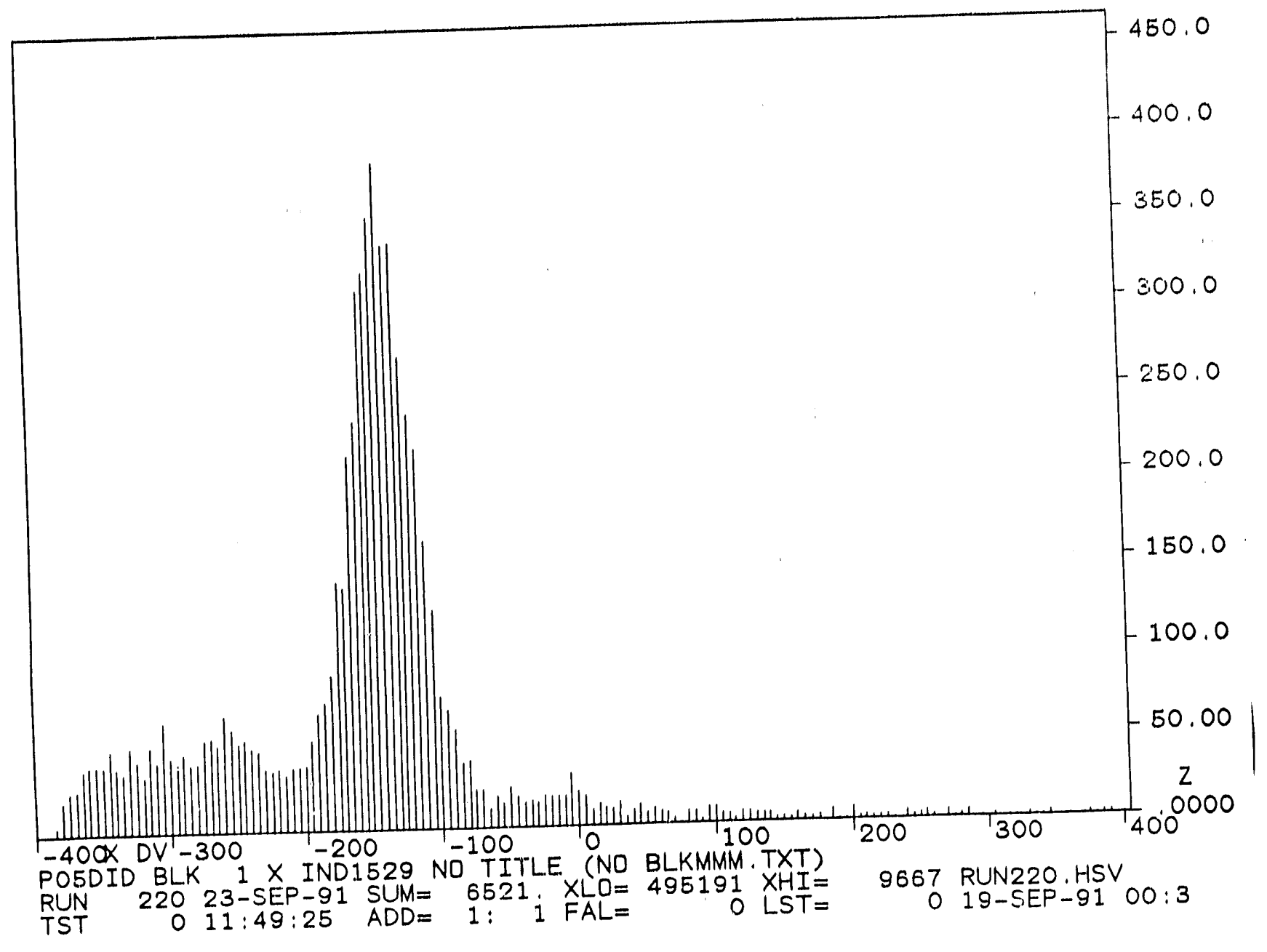

Figure 12. A proton identification spectrum for one of the phoswich detectors from the data in figure 11 . 


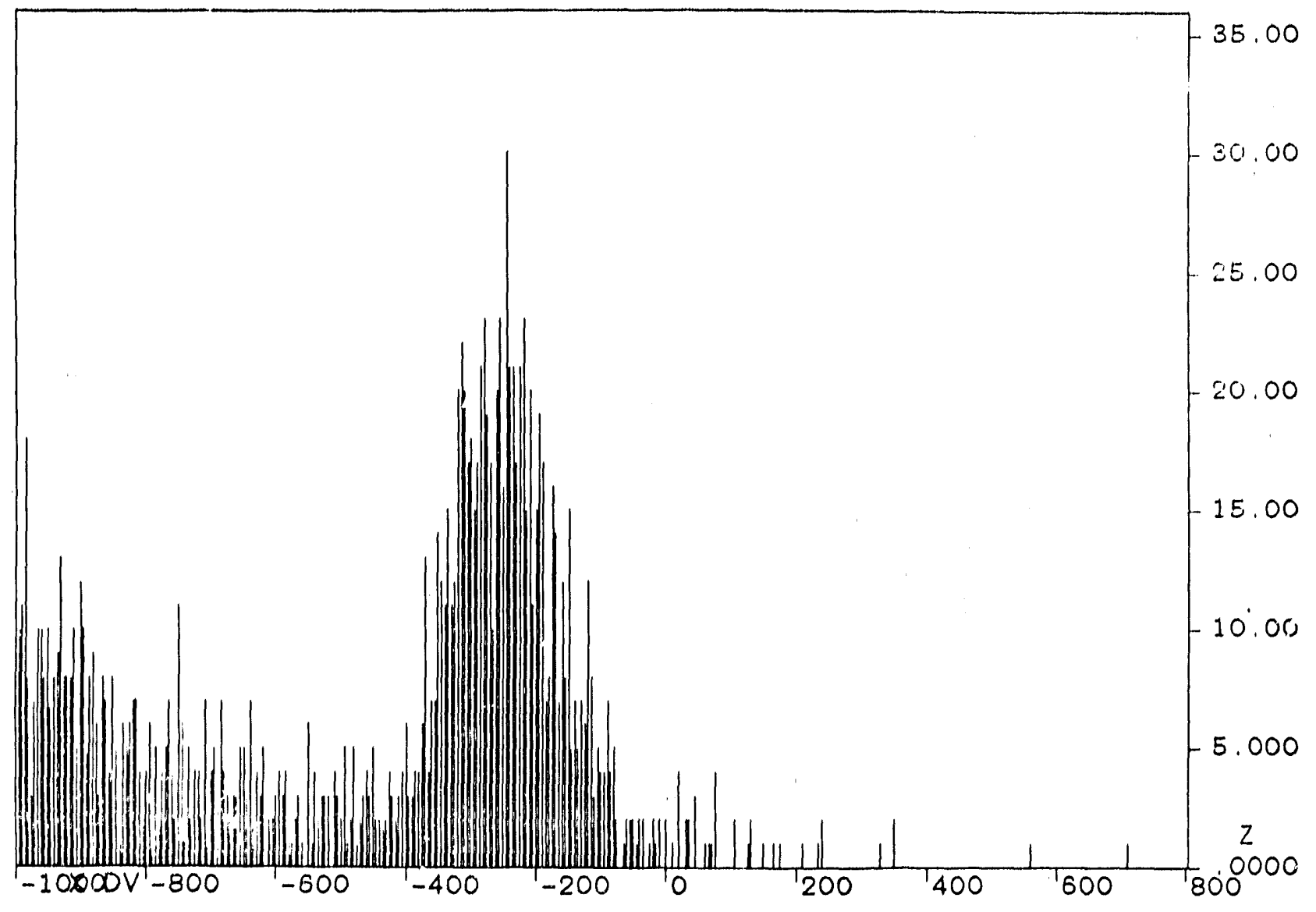

Figure 13. A proton identification spectrum for one of the nucleon detector bars from the same data set as shown in figure 11. 


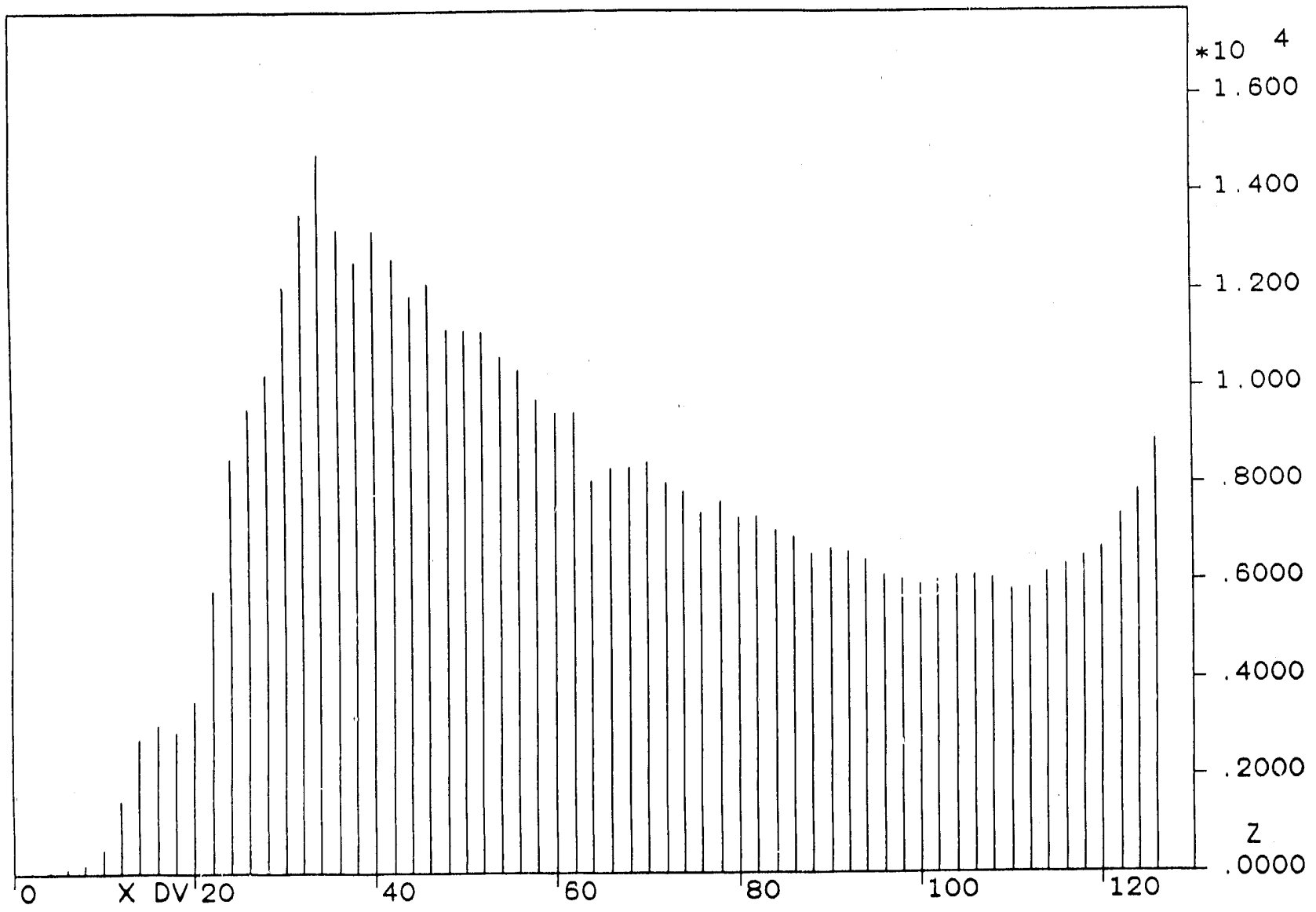

Figure 14. Plot of the scaler values for the photon tagger detector elements. The highest energy photons are produced when the stored electrons lose the greatest energy interacting with the laser photons. These electrons strike the detectors shown at the left side of the plot. Steps in the left edge are due to the fact that more than one laser output line is incident on the stored electrons. 
protons and neutrons were detected in an array of plastic scintillators. The experiment was the first use of the University of Virginia nucleon detector array and was valuable experience for its use in the ${ }^{3} \mathrm{He}(\gamma, \mathrm{NN})$ experiment at LEGS.

For the past year data have been analyzed by Cole Smith of the University of Virginia and partial, preliminary results are now available. The preliminary two-body yield is slightly more than three times the free $D(\pi, p p)$ yield or in other words, close to the number of possible pn pairs in ${ }^{4} \mathrm{He}$. The data show excess cross section at large missing momenta which is correlated with high missing energies where three- and four-body absorption dominates. An abstract which is included in the section on publications has been submitted to the Few Body XIII conference.

\section{CEBAF}

CEBAF is scheduled to begin operations in the second quarter of 1995 for Hall B which will contain the CEBAF Large Acceptance Spectrometer (CLAS) and toward which our CEBAF work is directed. We are currently coauthors of seven approved or conditionally approved proposals in Hall $B$ and Sealock is spokesman of one of them (Electroexcitation of the Delta in Nuclei). Thus prototyping work for the main detector elements of the CLAS is ongoing and construction will begin soon. We have committed ourselves to participating in the construction of the Electron/Gamma/Neutron (EGN) forward calorimeter, photon beam instrumentation and certain general beamline instrumentation. In the past year there has been active work on the EGN prototype and its final design, prototype detector elements for the photon tagger focal plane and a pair spectrometer for photon beam monitoring.

A prototype of the EGN detector was constructed in early 1990, was tested at the Brookhaven AGS test facility and is now being continually tested at CEBAF with cosmic rays. Those tests and others have raised questions about the light readout scheme. The prototype uses slabs of green waveshif ter placed near the ends of the scintillators to redirect the light at a $90^{\circ}$ angle. Disadvantages of this method are that photocathodes are less efficient for blue light than green, green fluors typically have longer decay constants than blue fluors and the waveshifter absorption spectrum isn't perfectly matched to the blue emission spectrum, leading to an apparent reduced attenuation length of the scintillator. We have made some measurements of decay constants for waveshifter material and found that the fastest commercially available green fluor decays in 4 nsec compared to $2-3 \mathrm{nsec}$ for standard blue fluors. An alternative has been proposed, namely optical fiber bundle light pipes glued to the ends of the scintillators. Tests of this scheme are underway. Early results indicate that this method gives twice as many photoelectrons when as little as $10 \%$ of the end of the scintillator is covered by fibers. 
The photon tagger focal plane is about 11 meters long and therefore requires a large number of individual detector elements, particularly if good energy resolution is to be achieved. In an effort to reduce costs it was proposed by Sealock that the Philips XP4702, 64 channel photomultiplier could be used with waveshifting fiber readout of the focal plane scintillators. This would reduce the number of high voltage channels and the number of photomultipliers by 64 . Several prototype detectors were constructed using different scintillators, waveshifters and reflectors. The scintillators were machined as rectangular solids, typically $4 \times 8 \times 50 \mathrm{~mm}^{3}$, and a single $1 \mathrm{~mm}$ diameter waveshifting fiber was glued into a groove in the scintillator. Diffuse reflectors such as teflon tape or white paint were put on the outside of the detector. Tests showed that this method easily gives enough photoelectrons (about 15 ) to detect the passage of minimum ionizing particles with essentially $100 \%$ efficiency. In addition a fiber readout is naturally flexible so that the phototube is easily removed from the region of the vacuum window where there is little space and the possibility of damage from a rupture.

Unfortunately, tests by the CEBAF detector group showed that there was unacceptable cross talk between the channels of the Philips tube. Meanwhile very inexpensive, side coupled phototubes, e.g. the Burle $931 \mathrm{~B}$, were being evaluated. The value of a flexible readout has been recognized. To that end, different fiber readout schemes are being tested. Since a 931 phototube doesn't require a tiny light input source, like the Philips tube does, a bundle of fibers can be used. Therefore we have tried multiple clear optical fibers glued to the end of a prototype detector. Our first version gave 40 photoelectrons and timing resolution of 500 psec FWHM. Although the light yield is lower than for a conventional light pipe it is quite adequate and the flexibility might more than compensate. Further tests and optimization are underway. A final decision on light pipe design will be made by the end of 1991.

We have been involved in the Photon Tagger group of Hall B at Cebaf since the equipment development started several years ago. Thornton has agreed in a Memorandum of Understanding with the CEBAF management that he will be responsible for the construction of a pair spectrometer to be used as a relative photon intensity monitor for the photon beam. A general design has been developed, and we are now looking for a suitable magnet. The ideal magnet would be of the $\mathrm{H}$ type. We have found one in End Station A at SLAC, but because of current efforts to develop End Station A into a facility for $50 \mathrm{GeV}$ nuclear and particle physics experiments, it is unlikely that we will be able to obtain that magnet. We will continue our search for an $\mathrm{H}$ or a $\mathrm{C}$ magnet, but we must find a suitable magnet during 1992. We will then design a detection system, and help Cebaf obtain vacuum equipment, power supplies, etc. 


\section{Waveshifting Optical Fiber Applications}

About a year ago Sealock and Oscar Rondon-Aramayo of the University of Virginia, began studies of novel applications of waveshifting optical fibers. The project that this section describes was intended as a proof of principle study of a new concept for medium energy neutron polarimetry. We have been motivated by a desire to measure form factors of the neutron at CEBAF. The following paragraphs describe hardware and software tests of the key ideas. Equipment has been purchased by means of an NSF Small Grant for Exploratory Research while the present grant has supported a number of part time and summer undergraduate students that have worked on this project. A prototype detector and a general purpose data acquisition system for optical fiber arrays, currently' under construction, are described. In the course of our work other applications of waveshifting fibers coupled to scintillators have been conceived. These are briefly described.

It has been demonstrated that it is feasible to use devices with thousands of optical fibers with individual pulse height measurements [11]. Also it has been shown that a waveshifting optical fiber can be used as a tiny readout for a larger volume of scintillator [12]. Thus it should be possible to combine scintillators, waveshifting fibers and multielement light detection devices so that a useful volume of neutron detector can have the spatial resolution required to track recoil protons from medium energy neutrons. With such a device one could measure neutron polarization with much greater efficiency than for the present double scattering devices (at most $0.27 \%$ [13]). Monte Carlo studies of a hypothetical detector indicate that this is true. Our proposed detector consists of 1800 scintillator bars each with a single fiber for light output. The bars are arranged as a stack of 20 hodoscopes with two planes of 45 bars each. The bars have dimensions of $1 \times 1 \times 45 \mathrm{~cm}^{3}$ and the fiber diameter is $1 \mathrm{~mm}$. Several planes of solid scintillator are interspersed in the tracking volume for timing measurements and vetoing incident charged particles. Light detection could be done either by an array of photodiodes or a chain of image intensifiers coupled to a Charge Coupled Device (CCD) camera.

A primary goal of this work was to measure the efficiency of the proposed detector element for detecting recoil protons. In the past year we have studied a great many combinations of liquid and solid scintillator, waveshifting fiber and reflector. Solid scintillators are preferred for convenience while liquid scintillators are better for polarimetry because they can have twice as many' hydrogen nuclei per carbon nucleus. We find that the best performance is obtained with a scintillator that emits ultraviolet light which is absorbed by a blue waveshifting fiber. The best reflector for the UV light is teflon tape. Blue waveshifters give better results than green waveshifters because photomultiplier photocathodes are about twice as efficient for blue light as for green light. Also the 
blue fluor has a shorter decay constant which gives better timing resolution.

A summary of our best results follows. All measurements were made with Phillips XP2262 photomultipliers with a bialkali photocathode and standard NIM and CAMAC electronics. Minimum ionizing particles from either cosmic rays or a ${ }^{90} \mathrm{Sr}$ source were used. The highest yield for a prototype detector element was 22 photoelectrons. The detector dimensions were $5 \times 10 \times 70 \mathrm{~mm}^{3}$ and it was made from Bicron Corporation BC422 plastic scintillator. A $1 \mathrm{~mm}$ diameter, $57 \mathrm{~cm}$ long, Bicron BCF 10 blue waveshifting fiber was glued into a groove and then the detector was wrapped in teflon tape. Prototype liquid scintillator detectors were made from rectangular cross section tubes of aluminized mylar filled with Bicron BC517H scintillator. A Bicron BCF91 green waveshifting fiber was centered in the tube. The yield was 7.5 photoelectrons. Liquid scintillators that emit in the UV can be obtained which would allow use of blue fibers. When this yield is multiplied by two to account for greater photocathode efficiency and by another factor of two to account for the greater energy loss of recoiling protons, inefficiency is of no concern.

A model of the detector has been coded to simulate the operation of the device using Monte Carlo techniques. At the present time, the program simulates the interactions of neutrons with hydrogen nuclei in the scintillator, based on the SAID [14] code, to generate the yields of recoil protons as a function of the incident neutron kinetic energy and the neutron-proton angle. The leftright asymmetry is also obtained from the same code from the differential cross section and analyzing power for $n-p$ elastic scattering. The recoil protons are tracked through the detector and their energy loss per cell is computed using the Bethe-Bloch equation. Light yield outputs are also calculated, based on $d e / d x$ and the properties of the scintillator. To illustrate the use of this simulation in Fig. 15 we show plots for the recoil proton lab angles and the distribution of analyzing powers per event. The detector had 1800 bars arranged in 20 layers of $45 x$ by $45 y 1 \mathrm{~cm}$ square bars. The histograms with error bars correspond to good proton tracks that stop in the detector; the plain histograms indicate good tracks that leave the detector. The incident neutron energy was $533 \mathrm{MeV}$ The calculated efficiency, the ratio of the number of neutrons that scatter and produce a recoil proton fully contained within the detector to the number of incident neutrons, is $1.1 \%$. This is more than 3 times greater than the efficiency of conventional detectors [13]. The efficiency of our device could be approximately doubled by doubling the thickness since the hardware cost to read out 3600 fibers would not be excessive. Also the small size of our device makes it much easier to shield or to move than conventional polarimeters. Further Monte Carlo studies will allow us to optimize the detector shape and cell size. The light yield of the detector elements and the Monte Carlo studies indicate that our concept is feasible and that only questions of practicality remain. Therefore we are now studying light detection schemes and have built a prototype tracking detector. 


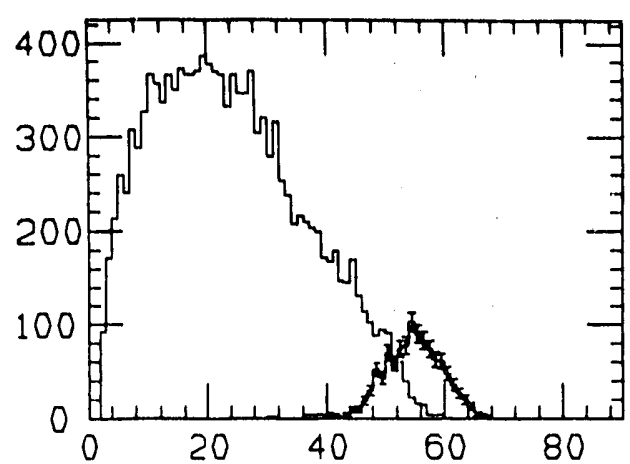

A. Recoll proton Lab angle

$T_{n}: 160 \mathrm{MeV}$

$Q^{2}: 0.300[\mathrm{GeV} / \mathrm{c}]^{2}$

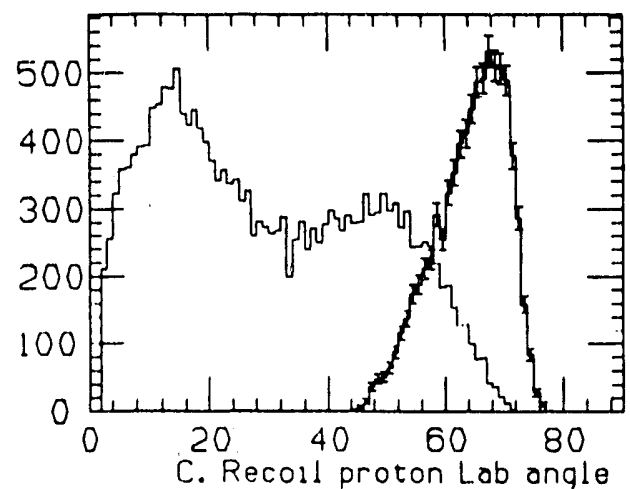

$T_{n}: 533 \mathrm{MeV}$

$0^{2}: \quad 1.0[\mathrm{GeV} / \mathrm{c}]^{2}$

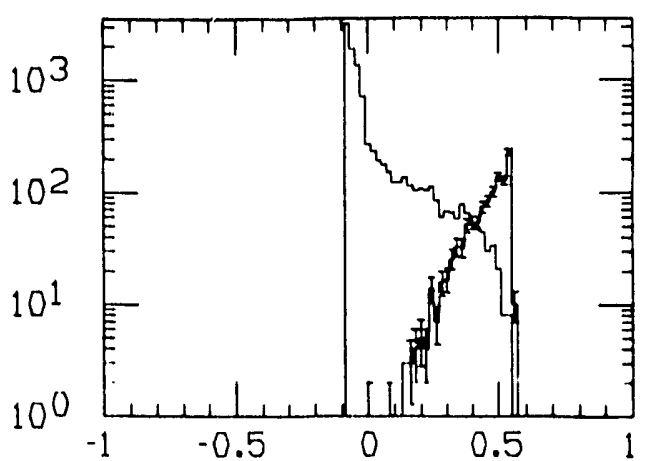

B. Analyzing power dist.

$T_{n}: 160 \mathrm{MeV}$

$Q^{2}: \quad 0.300[\mathrm{GeV} / \mathrm{c}]^{2}$

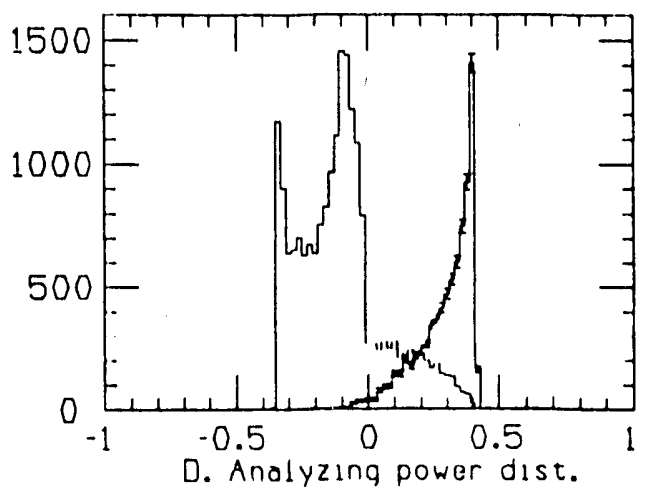

$T_{n}: 533 \mathrm{MeV}$

$Q^{2}: \quad 1.0(\mathrm{GeV} / \mathrm{C})^{2}$

Figure 15. Monte Carlo simulations of the neutron polarimetry performance of a scintillator and waveshifting fiber tracking detector. The histograms with statistical error bars indicate the numbers of recoil protons, out of 40,000 neutron interactions, whose tracks are usefully long and completely contained within the detector volume. The plain histograms indicate recoil protons that escape the detector. 
The small size and simple construction of photodiodes makes them attractive readout devices. We have studied the response of three photodiodes (Centronic OSD-5T and Hammamatsu 1336-18BK and 1087-03) and two avalanche photodiodes (APD's) (Hammamatsu S2381 and RCA 30902E). To this effect, several amplifier circuits were built, based on both inexpensive commercial amplifiers and moderately priced low noise amplifiers. The best results were obtained with the LeCroy TRA 1000 amplifier, which has a rise time of $25 \mathrm{~ns}$ and input noise of about $2 \mathrm{pA} \sqrt{\mathrm{Hz}}$. This noise at the frequency of the amplifier bandwidth (14 Mhz) is equivalent to $7.5 \mathrm{Na}$, so for the $130 \mathrm{~ns}$ wide PD pulses, it is equivalent to a charge of 6000 electrons. Therefore, for the APD's, which have gains of - 150 and quantum efficiencies of $50 \%$ for the green light emitted by the waveshifting fibers, we expect that pulses of about 160 photons can be detected with a signal-to-noise ratio of 2 . For the ordinary PD's, about 24000 photons would be needed. In actual tests, the complete circuit noise for the TRA 1000 for $\mathrm{a} \sim 2: 1 \mathrm{~s}-\mathrm{n}$ ratio using light from pulsed green LED's, and the Centronics OSD-5T photodiode, was higher, requiring $5 \times 10^{4}$ photons to give a detectable signal, or more than twice the estimated noise. The implication is that, given the much greater cost per channel for APD based detectors (10 times) than for ordinary PD's, and in view of the high light flux required to use the inexpensive PD's, for detectors with a large number of channels $(>200)$ it may pay to amplify the light from the fibers - rather than the electronic signals - using an image intensifier with a gain of $\sim 10^{4}$, unless a significantly quieter low cost amplifier is available.

We have constructed a small prototype tracking detector that we will use as a test device for detector elements, light detection schemes and particle tracking. The design allows interchangability of detector elements and the whole array can be used with any method for detecting fiber light. Figure 16 shows the arrangement of detector elements and trigger planes. The individual detector elements have dimensions of $5 \times 10 \times 70 \mathrm{~mm}^{3}$. Seven elements make up one layer and two layers arranged at right angles form a hodoscope. Seven hodoscopes are stacked to make a detector with 98 fiber outputs and a volume of $7 \times 7 \times 7 \mathrm{~cm}^{3}$ containing 343 virtual $1 \mathrm{~cm}^{3}$ cells. Two types of detector elements are indicated - plastic scintillator bars with waveshif ting fibers glued into grooves and liquid scintillator cells with fibers in the center of each cell. Other types of elements may be substituted. Four trigger planes of plastic scintillator with light guides and photomultipliers are shown. The outer trigger planes can indicate charged particles that are incident or recoil particles that escape while the inner trigger planes will give timing and trigger pulses for recoil particles. The ideal neutron event will produce a recoil proton that makes detectable light in all the cells that it crosses as well as the two internal, but not the external, trigger planes. Cosmic rays and accelerator produced neutrons will be used to test the detector. A practical detector would be similar to the prototype but would have several thousand elements and could be of most any shape. 

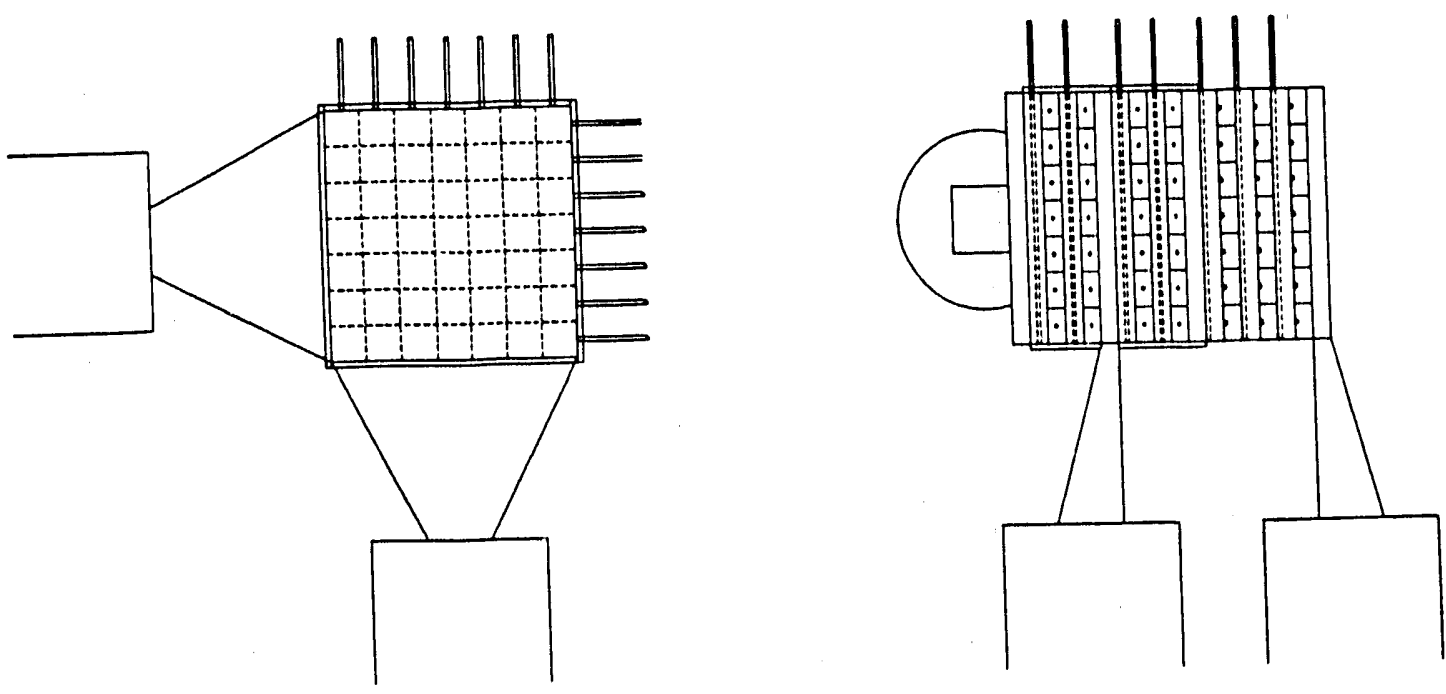

Figure 16. A prototype tracking detector using scintillator bars with waveshifting fiber readout. The device has 98 scintillators arranged as 7 hodoscopes and 4 trigger planes
photomultipliers. Fiber light is detected by an intensified CCD camera. 
We have purchased the major components of a light detection and data acquisition system. Light from the fibers will be amplified and detected by two image intensifiers coupled to a Charge Coupled Device camera which will be read out by a Camac transient digitizer. The intensifiers and camera are coupled by fiber optic couplers to maintain spatial information. The first intensifier is a high gain, microchannel plate intensifier with $18 \mathrm{~mm}$ active diameter. It can be gated off while the camera is being read out. The second intensifier is a low gain, electrostatic intensifier that reduces the image to match the dimensions of the CCD sensor. The total gain is greater than $10^{6}$ so that individual photons can be detected. There is a fiber optic taper at the input of the first intensifier that can accommodate $1001 \mathrm{~mm}$ fibers and reduce the image to the diameter of the first intensifier. This input coupler is removable, allowing us to upgrade the intensifier chain to handle 2000 fibers by adding a large diameter, electrostatic, demagnifying intensifier.

The CCD camera is an EG\&G Reticon MC9128 camera with a $128 \times 128$ pixel format and a maximum rate of 380 frames per second. The camera can be cleared frequently to avoid buildup of dark charge between events. Camera output will be digitized by a DSP Technologies model 2008A Camac transient digitizer. The digitizer memory is tuansferred to a $386 \mathrm{PC}$ via a Jorway SCSI Camac crate controller. Since the camera and digitizer procluce $16 \mathrm{k}$ bytes of data per event the relatively high speed of the SCSI bus and a large hard disk are important. A data acquisition program has been written that allows us to read and store the digitizer output as well as to issue CAMAC control commands. Electronic circuitry to control the camera has been partially built.

In the process of developing the neutron polarimeter we have thought of other devices that use waveshifting fibers to read out scintillators. These are a large volume, medium energy neutron detector and a flexible light pipe scheme for the CEBAF CLAS photon tagger detector array. The latter is described in the section on our contributions to CEBAF.

It has been reported that scintillating fibers can give better timing resolution than bulk scintillators [15]. This has been explained in terms of reduced path length dispersion of the scintillation photons as they travel to a photomultiplier. We are trying to take advantage of this by using an array of waveshifting fibers to absorb, reemit and transport the light from a large volume of liquid scintillator. We have constructed and tested a tank containing liquid scintillator and a cylinder of waveshifting fibers. The interior dimensions were $10 \times 10 \times 160 \mathrm{~cm}^{3}$ in order to compare the performance with existing solid plastic neutron detector bars. The potential advantages are that in large volumes liquid scintillator is significantly cheaper, it can have twice as many protons per carbon nucleus as plastics and timing resolution may be better. Preliminary results indicate that the light yield is surprisingly high but the timing is poor. We attribute the poor timing to the fact that the decay constant of the fluor in the fibers we used was $12 \mathrm{nsec}$ which resulted in a very slow rising pulse. Substituting a UV liquid scintillator and fast decaying blue fiber is straightforward. We now 
have the experience to design a detector using optimum materials and methods that should achieve superior performance at lower cost for large volume neutron detectors.

We regard our progress so far as only the beginning of long term development work on position sensitive neutral particle detectors for both basic and applied purposes. Since starting this project there has been improvement in the commercially available scintillating and waveshifting optical fibers. Also it is now possible to purchase a 4096 pixel CCD camera that can operate at 25,000 frames per second. Further developments in commercial hardware will make detectors using thousands of fibers more practical. In the near future we will complete and test the 98 channel prototype tracking detector and continue design studies for a full size neutron polarimeter.

\section{Education of Students}

In the past year we have had several students working with us part time during the academic year or full time in the summer. Their projects have been largely hands-on laboratory work related to applications of optical fibers. We find that today's students are more likely to be skilled in the use of computers than in laboratory techniques. Thus, equipment development projects are particularly valuable experience.

A graduate, Brian Milbrath, worked at LEGS on preparations for measurements of Compton scattering from the proton. Another graduate student, Bifford Lyons, worked with Sealock full tinie in the summer of 1991. He was half supported by another DOE grant since he worked on waveshifting fiber applications jointly with Oscar Rondon-A ramayo. He worked on data acquisition software and an electronic circuit that are both required to read and store the output of a CCD camera.

During the 1990/1991 academic year we supported two undergraduates, Rose Finn and David Jones, who worked about 10 hours a week each, excluding exam weeks and breaks. Their projects included machining of scintillators, construction of electronics, making drawings with AutoCad, and Monte Carlo simulations of detectors. A third student, Mark Lesko, was supported by another DOE grant but spent about half of his time constructing electronics to control the CCD camera for the waveshifting fiber prototype tracking detector. During the summer of 1991 Sealock supervised a student, Adam Shively of North Park College, in the University of Virginia, Research Experiences for Undergraduates program. His project was to construct and test a large tank containing liquid scintillator with a bundle of waveshifting fibers for readout to be used for neutron detection. His work was awarded top honors by the director of the REU program, Richard Lindgren, and he will present his results at the Sixth National Conference on Undergraduate Research in 1992. 


\section{References}

1. D. H. Dowell et al., Nucl. Instrum. Methods A286, 183 (1989).

2. G. S. Blanpied et al., Phys. Rev. Lett. 67, 1206 (1991).

3. P. Wilhelm, W. Leidemann and H. Arenhoeval (private communication), extensions of calculations in Few Body Syst. 3, 111 (1988).

4. E. DeSanctis et al., Phys. Rev. C34, 413 (1986).

5. P. Levi Sandri et al., Phys. Rev. C39, 1701 (1989).

6. F. F. Lui, Phys. Rev. 138, B1443 (1965).

7. S. Nozawa, B. Blanklieder and T.-S. H. Lee, Nucl. Phys. A513, 459 (1990).

8. R.A. Arndt, R. L. Workman, Z. Li and L. D. Roper, Phys. Rev. C42, 1853 (1990).

9. H. Genzel, E. Hilger, G. Knop, H. Kemen and R. Wedemeyer, Z. Physik 268, 43 (1974).

10. D. Dougan, V. Ramsey and W Stiefler, Z. Physik A276, 155 (1976).

11. E. Ansorge et al., Nucl. Instrum. Meth. A265, 33 (1989).

12. H. Blumenfeld, M. Bourdinaud and J. C. Thevenin, Nucl. Instrum. Meth. A257, 603 (1987).

13. R. Madey et al., IEEE Trans. Nucl. Sci. 36, 231 (1989).

14. Richard A. Arndt, John S. Hyslop and L. David Roper, Phys. Rev. D35, 128 (1987).

15. M. Kuhlen et al., Nucl. Instrum. Meth. A301, 223 (1991). 


\section{Publications $\star$}

A. Journals and Proceedings

1. "Beam-Polarization Observables in $D(\vec{\gamma}, p) n$ and the Nuclear Tensor Force", G.S. Blanpied, M. Blecher, A. Caracappa, C. Djalali, M-A. Duval, G. Giordano, S. Hoblit, O.C. Kistner, G. Matone, L. Miceli, W.K. Mize, B.M. Preedom, A.M. Sandorfi, C. Schaerf, R.M. Sealock, C.E. Thorn, S.T. Thornton, K. Vaziri and C.S. Whisnant, Phl's, Rev. Lett. 67, 1206 (1991).

2. "Longitudinal and Transverse Response Functions in ${ }^{56} \mathrm{Fe}(\mathrm{e}, \mathrm{e})$ At Momentum Transfer Near $1 \mathrm{GeV} / \mathrm{c}^{\prime}$, J.P. Chen, Z.E. Meziani, D. Beck, G. Boyd, L.M. Chinitz, D.B. Day, L.C. Dennis, G. Dodge, B.W. Filippone, K.L. Giovanetti, J. Jourdan, K.IV. Kemper, T. Koh, W. Lorenzon, J.S. McCarthy, R.D. McKeown, R.G. Milner, R.C. Minehart, J. Morgenstern, J. Mougey, D.H. Potterveld, O.A. Rondon-Aramayo, R.M. Sealock, L.C. Smith, S.T. Thornton, R.C. Walker, and C. Woodward, Phys. Rev. Le'l. $66,1283(1991)$.

3. "Elemental variations in the Germinating Fungus Phytophthora palmivora", A. Mazzolini, B.R. Grant, R.M. Sealock and G.J.F. Legge, Nucl. Instr. and Meth. B54, 151 (1991).

4. "A Study of the Photon Decay Modes of the Giant Dipole Resonance in Even-A Nd Isotopes", S. D. Hoblit and A. M. Nathan, Phys. Rev. C, to be published.

B. Abstracts

1. "Simulation of a Waveshifting Fiber-in-Scintillator (FIS) Neutron Detector/Polarimeter", O. A. Rondon-Aramayo and R. M. Sealock, Bull. Am. Phys. Soc. 36, 1301 (1991).

2. "Measurements of the Transverse and Longitudinal Response Functions for ${ }^{3} \mathrm{He}$ and "He at q near $1 \mathrm{GeV} / \mathrm{c}$ ", J.P. Chen, L. Chinitz, D. Day, K. Giovanetti, J.S McCarthy', R.C. Minehart, O. Rondon-Aramayo, R.M. Sealock, L.C. Smith, S.T. I hornton, Z.E. Meziani, G. Dodge, D. Beck, G. Boyd, B.W. Filipone, J. Jourdan, R.D. McKeown, R.G. Milner, D. Potterveld, R. Walker, C. Woodward, L.C. Dennis, K.W. Kemper, W. Lorenzon, I. Sick, J. Morgenstern, J. Mougey, Bull. Am. Phy's. Soc. 36, 2125 (1991).

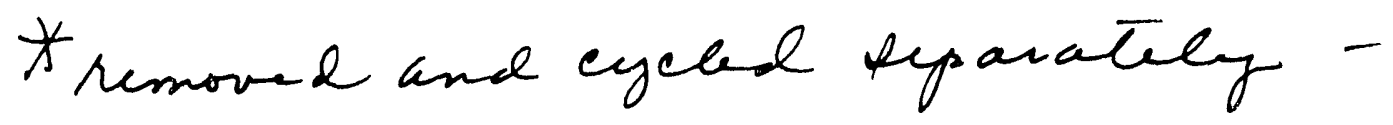



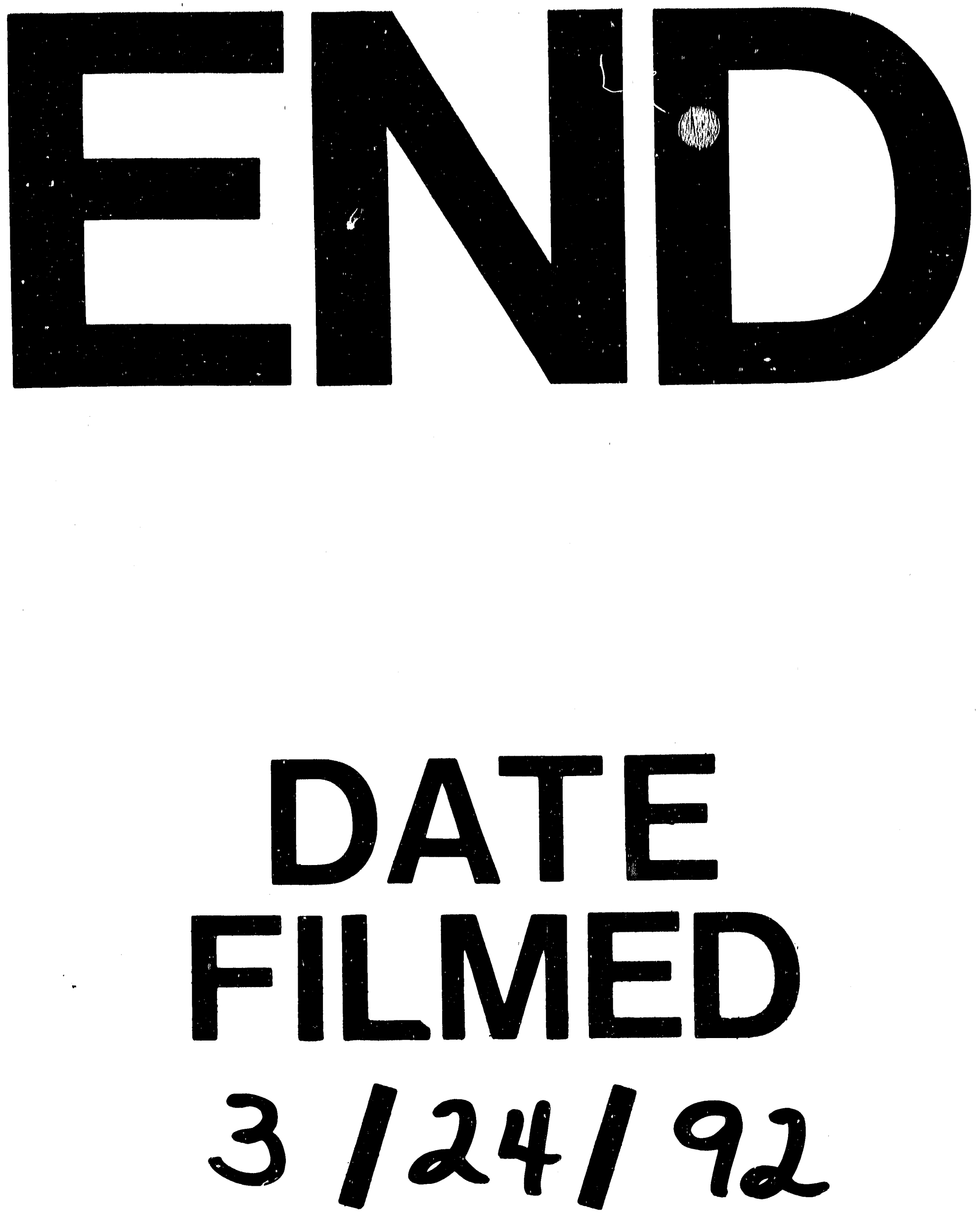
